\title{
The electromagnetic decays of the charmed and bottom baryons in chiral perturbation theory
}

\author{
Nan Jiang* Xiao-Lin Chen * $^{*}$ and Shi-Lin Zhu \\ ${ }^{1}$ School of Physics and State Key Laboratory of Nuclear Physics and Technology, Peking University, Beijing 100871, China \\ ${ }^{2}$ Collaborative Innovation Center of Quantum Matter, Beijing 100871, China
}

\begin{abstract}
We have investigated the electromagnetic decays of the antitriplet and sextet charmed baryon systems with $J^{P}=\frac{1}{2}^{+}, \frac{3}{2}^{+}$in the framework of the heavy baryon chiral perturbation theory. We first construct the chiral Lagrangians at $O\left(p^{2}\right)$ and $O\left(p^{3}\right)$. Then we calculate the electromagnetic (EM) decay amplitudes of the charmed baryon systems up to $O\left(p^{3}\right)$. With the help of the quark model, we estimate the low energy constants. The numerical results of the EM decay widths show good convergence of the chiral expansion. We notice that the two neutral EM decay processes $\Xi_{c}^{\prime 0} \rightarrow \gamma+\Xi_{c}^{0}$ and $\Xi_{c}^{* \prime 0} \rightarrow \gamma+\Xi_{c}^{0}$ are strongly suppressed by the $\mathrm{SU}(3)$ U-spin flavor symmetry. With the same formalism, we also estimate the EM decay widths of the bottomed baryons. The EM decay widths of the heavy baryons may be measured at facilities such as LHCb and JPARC. The explicit chiral structures of the heavy baryon decay amplitudes derived in this work may be useful to the possible chiral extrapolations of the future lattice simulations of these EM decay amplitudes.
\end{abstract}

PACS numbers: 12.39.Fe, 14.20.Lq, 13.30.-a, 13.40.Hq

\section{INTRODUCTION}

The low energy behavior of quantum chromodynamics (QCD) is highly nonperturbative and complicated. The study of the hadron properties helps us explore the infrared structure of QCD. In the past decades many heavy baryons were discovered experimentally [1-3].

The heavy flavored baryon systems containing a single heavy quark are particularly interesting. In the heavy quark limit, there exists the additional heavy quark spin and flavor symmetry when the heavy quark mass goes to infinity. The strong dynamics within the heavy baryon is governed by the two light quarks while the heavy quark acts as a color source and the spectator. The physical observables can be expanded in terms of $1 / m_{Q}$ where $m_{Q}$ is the heavy quark mass.

In this work, we focus on the heavy baryons containing a single charm or bottom quark and investigate their electromagnetic (EM) decays. The heavy baryon ground states contain the spin- $\frac{1}{2}$ sextet, antitriplet and the spin- $\frac{3}{2}$ sextet. For brevity, we refer to them as the $6, \overline{3}$ and $6^{*}$ dimensional baryons in the following. We will explore the property of the electromagnetic transitions between the heavy baryon multiplets.

In literature, various theoretical approaches were employed to study the magnetic moments and EM decays of the light baryons [4 20]. We have performed a systematic study of the chiral corrections to the heavy baryon mass and axial charge in Ref. 21]. In this work, we will investigate the electromagnetic decay width of the antitriplet and sextet heavy baryon systems in the framework of heavy baryon chiral perturbation theory (HBChPT). We truncate the chiral expansion at $O\left(p^{3}\right)$. The tree-level electromagnetic decay amplitude appears at $O\left(p^{2}\right)$ and $O\left(p^{3}\right)$. We include the chiral loop corrections up to $O\left(p^{3}\right)$. The recoil corrections in terms of $\frac{1}{M}$ appear at $O\left(p^{4}\right)$ or even high order. As will be seen below, our results show good convergence of the chiral expansion.

There are twelve possible EM decay channels. Because of the constraint of flavor structure, only ten channels arise at the leading order. At $O\left(p^{3}\right)$, there appear six additional EM interaction low energy constants involving the 6 -dimensional and $6^{*}$-dimensional baryons at the tree level. In contrast, the loop contributions appear at $O\left(p^{3}\right)$ and contribute to all twelve channels.

The EM width is proportional to the cubic of the photon momentum, which is roughly equivalent to the mass difference of the initial and final states. The average value of the mass difference is $127 \mathrm{MeV}$ between the $\overline{3}$ and 6-dimensional charmed baryons, $67 \mathrm{MeV}$ between the 6 and $6^{*}$-dimensional baryons, $194 \mathrm{MeV}$ between $\overline{3}$ and $6^{*}$ dimensional baryons. In the calculation of the EM decay widths, we use the experimental values of the charmed baryon masses as input to derive the photon momentum. The EM decay widths of different channels differ greatly,

\footnotetext{
*Electronic address: Jiangn@pku.edu.cn

${ }^{\dagger}$ Electronic address: chenxl@pku.edu.cn

${ }^{\ddagger}$ Electronic address: zhusl@pku.edu.cn
} 
mainly due to the large difference of the photon momentum. In the appendix, we also extend the same formalism to estimate the EM widths of the bottomed baryons.

This paper is organized as follows. In Sec. II, we introduce the effective chiral Lagrangians up to $O\left(p^{3}\right)$. In Sec. III, we calculate the EM decay amplitude and width order by order. In Sec. IV, we determine the low energy constants (LECs) using the quark model. In Sec. V, we discuss the numerical results and summarize. We collect some useful formulae and the numerical results of the EM decay of the bottom baryons in the appendix.

\section{THE CHIRAL LAGRANGIANS}

\section{A. The Leading Order Chiral Lagrangians}

The three light quarks $u, d, s$ form the fundamental representation under the flavor SU(3) transformation. The heavy baryons can be classified into multiplets in flavor SU(3) space. Now we concentrate on the ground states of the charmed baryon. When the two light quarks are in the flavor antitriplet, the quantum number of the heavy baryon is $J^{P}=\frac{1}{2}^{+}$. When the two light quarks are in the symmetric flavor sextet, the quantum number of the heavy baryon can be either $J^{P}=\frac{1}{2}^{+}$or $\frac{3}{2}^{+}$.

In this work, we investigate the heavy baryon systems employing the Chiral Perturbation Theory (ChPT) [22], which provides a systematic expansion of the physical observables in terms of small momentum $p$ and the mass of Goldstone bosons $m$. ChPT has been widely used to study the low energy hadron interaction. In the early stage, it was employed to study the purely mesonic system [23, 24]. Later it was extended to discuss the baryon-meson system 25 28]. In order to deal with the heavy baryon system, the heavy baryon chiral perturbation theory (HBChPT) was developed 32 35], which provides a convenient framework to make a dual expansion in terms of both the small momentum and $\frac{1}{M}$, where $M$ is the heavy baryon mass.

In the flavor space, the pseudoscalar meson fields and spin- $\frac{1}{2}$ baryon multiplets are defined as follows

$$
\begin{gathered}
\phi=\left(\begin{array}{ccc}
\pi^{0}+\frac{1}{\sqrt{3}} \eta & \sqrt{2} \pi^{+} & \sqrt{2} K^{+} \\
\sqrt{2} \pi^{-} & -\pi^{0}+\frac{1}{\sqrt{3}} \eta & \sqrt{2} K^{0} \\
\sqrt{2} K^{-} & \sqrt{2} \bar{K}^{0} & -\frac{2}{\sqrt{3}} \eta
\end{array}\right) \\
B_{\overline{3}}=\left(\begin{array}{ccc}
0 & \Lambda_{c}^{+} & \Xi_{c}^{+} \\
-\Lambda_{c}^{+} & 0 & \Xi_{c}^{0} \\
-\Xi_{c}^{+} & -\Xi_{c}^{0} & 0
\end{array}\right), \quad B_{6}=\left(\begin{array}{ccc}
\Sigma_{c}^{++} & \frac{1}{\sqrt{2}} \Sigma_{c}^{+} & \frac{1}{\sqrt{2}} \Xi_{c}^{\prime+} \\
\frac{1}{\sqrt{2}} \Sigma_{c}^{+} & \Sigma_{c}^{0} & \frac{1}{\sqrt{2}} \Xi_{c}^{\prime 0} \\
\frac{1}{\sqrt{2}} \Xi_{c}^{\prime+} & \frac{1}{\sqrt{2}} \Xi_{c}^{\prime 0} & \Omega_{c}^{0}
\end{array}\right)
\end{gathered}
$$

The spin- $\frac{3}{2}$ baryons $B_{6}^{* \mu}$ are the so-called Rarita-Schwinger vector-spinor fields [36], which are similar to $B_{6}$. In the framework of ChPT, one can construct the Lagrangians order by order while the specific building blocks satisfying the chiral transformation are defined as follows [29, 30].

$$
\begin{aligned}
& U(x)=e^{\frac{i}{F_{0}} \phi(x)}, \quad u^{2}=U \\
& \Gamma_{\mu}=\frac{1}{2}\left(u^{\dagger} \partial_{\mu} u+u \partial_{\mu} u^{\dagger}\right) \\
& u_{\mu}=\frac{i}{2}\left(u^{\dagger} \partial_{\mu} u-u \partial_{\mu} u^{\dagger}\right) \\
& D_{\mu} B=\partial_{\mu} B+\Gamma_{\mu} B+B \Gamma_{\mu}^{T} \\
& \nabla_{\mu} U=\partial_{\mu} U-i r_{\mu} U+i U l_{\mu} \\
& r_{\mu}=l_{\mu}=-e Q \mathcal{A}_{\mu}, \quad Q=\left(\begin{array}{ccc}
\frac{2}{3} & 0 & 0 \\
0 & -\frac{1}{3} & 0 \\
0 & 0 & -\frac{1}{3}
\end{array}\right)
\end{aligned}
$$

The superscript $T$ denotes the transpose in the flavor space. The pion decay constant $F_{0} \approx 92.4 \mathrm{MeV}$. The pure meson Lagrangian in the lowest order $O\left(p^{2}\right)$ is

$$
\mathcal{L}^{(2)}=\frac{F_{0}^{2}}{4} \operatorname{Tr}\left[\nabla_{\mu} U\left(\nabla_{\mu} U\right)^{\dagger}\right]
$$


The leading order pseudoscalar meson and heavy baryon Lagrangians at $O\left(p^{1}\right) \operatorname{read}[37]$

$$
\begin{aligned}
\mathcal{L}_{0}^{(1)}= & \frac{1}{2} \operatorname{Tr}\left[\bar{B}_{\overline{3}}\left(i \not D-M_{\overline{3}}\right) B_{\overline{3}}\right]+\operatorname{Tr}\left[\bar{B}_{6}\left(i \not D-M_{6}\right) B_{6}\right] \\
& +\operatorname{Tr}\left\{\bar{B}_{6}^{* \mu}\left[-g_{\mu \nu}\left(i \not D-M_{6}^{*}\right)+i\left(\gamma_{\mu} D_{\nu}+\gamma_{\nu} D_{\mu}\right)-\gamma_{\mu}\left(i \not D+M_{6}^{*}\right) \gamma_{\nu}\right] B_{6}^{* \nu}\right\} \\
\mathcal{L}_{\text {int }}^{(1)}= & g_{1} \operatorname{Tr}\left(\bar{B}_{6} \psi \gamma_{5} B_{6}\right)+g_{2}\left[\operatorname{Tr}\left(\bar{B}_{6} \psi \gamma_{5} B_{\overline{3}}\right)+\text { h.c. }\right]+g_{3}\left[\operatorname{Tr}\left(\bar{B}_{6}^{* \mu} u_{\mu} B_{6}\right)+\text { h.c. }\right] \\
& +g_{4}\left[\operatorname{Tr}\left(\bar{B}_{6}^{* \mu} u_{\mu} B_{\overline{3}}\right)+\text { h.c. }\right]+g_{5} \operatorname{Tr}\left(\bar{B}_{6}^{* \nu} \psi \gamma_{5} B_{6 \nu}^{*}\right)+g_{6} \operatorname{Tr}\left(\bar{B}_{\overline{3}} \psi \gamma_{5} B_{\overline{3}}\right)
\end{aligned}
$$

where $\mathcal{L}_{0}^{(1)}$ and $\mathcal{L}_{\text {int }}^{(1)}$ are the free and interaction part. From the quark model and flavor SU(3) symmetry, the axial coupling constants $g_{6}=0, g_{1}=0.98, g_{1}=-\sqrt{\frac{8}{3}} g_{2}$ [37]. The heavy quark spin flavor symmetry leads to the following relations among these coupling constants, i.e., $g_{3}=\frac{\sqrt{3}}{2} g_{1}, g_{5}=-\frac{3}{2} g_{1}, g_{4}=-\sqrt{3} g_{2}$. Numerically, $g_{1}=0.98, g_{2}=-0.60, g_{5}=-1.47, g_{3}=0.85, g_{4}=1.04$.

In the framework of HBChPT, the baryon field $B$ is decomposed into the large component $\mathcal{N}$ and the small component $\mathcal{H}$. Their relationships are

$$
\begin{aligned}
& B=e^{-i M v \cdot x}(\mathcal{N}+\mathcal{H}) \\
& \mathcal{N}=e^{i M v \cdot x} \frac{1+\psi}{2} B, \quad \mathcal{H}=e^{i M v \cdot x} \frac{1-\psi}{2} B
\end{aligned}
$$

where $v^{\mu}$ is the on shell velocity. For the spin- $\frac{3}{2}$ baryon, the large component is denoted as $\mathcal{T}^{\mu}$. Such a decomposition is equivalent to separating the off-shell momentum $k$ from the whole momentum $p$ of the heavy baryon while the on shell part is $M v$. The reduced effective Lagrangian only relies on the large component. Omitting some details, the nonrelativistic Lagrangian in the heavy quark limit reads

$$
\begin{aligned}
\hat{\mathcal{L}}^{(1)}= & \frac{1}{2} \operatorname{Tr}\left[\overline{\mathcal{N}}_{\overline{3}}(i v \cdot D) \mathcal{N}_{\overline{3}}\right]+\operatorname{Tr}\left[\overline{\mathcal{N}}_{6}\left(i v \cdot D-\delta_{1}\right) \mathcal{N}_{6}\right]+\operatorname{Tr}\left\{\overline{\mathcal{T}}^{\rho}\left[-g_{\rho \sigma}\left(i v \cdot D-\delta_{2}\right)\right] \mathcal{T}^{\sigma}\right\} \\
& +2 g_{1} \operatorname{Tr}\left(\overline{\mathcal{N}}_{6} S \cdot u \mathcal{N}_{6}\right)+2 g_{2}\left[\operatorname{Tr}\left(\overline{\mathcal{N}}_{6} S \cdot u \mathcal{N}_{\overline{3}}\right)+\text { h.c. }\right]+g_{3}\left[\operatorname{Tr}\left(\overline{\mathcal{T}}^{\mu} u_{\mu} \mathcal{N}_{6}\right)+\text { h.c. }\right] \\
& +g_{4}\left[\operatorname{Tr}\left(\overline{\mathcal{T}}^{\mu} u_{\mu} \mathcal{N}_{\overline{3}}\right)+\text { h.c. }\right]+2 g_{5} \operatorname{Tr}\left(\overline{\mathcal{T}}^{\nu} S \cdot u \mathcal{T}_{\nu}\right)+2 g_{6} \operatorname{Tr}\left(\overline{\mathcal{N}}_{\overline{3}} S \cdot u \mathcal{N}_{\overline{3}}\right)
\end{aligned}
$$

The mass difference parameters are defined as $\delta_{1}=M_{6}-M_{\overline{3}}, \delta_{2}=M_{6^{*}}-M_{6}$. In the isospin symmetry limit, $\delta_{1}=126.52 \mathrm{MeV}, \delta_{2}=67.03 \mathrm{MeV}$. We have not kept the $\frac{1}{M}$ corrections to the Lagrangian because their contributions are of higher order.

\section{B. The higher order Lagrangians for the EM decays}

Considering the conservation of the electric charge and energy, there exist twelve electromagnetic decay channels, which can be classified into three types:

$B_{6} \rightarrow B_{\overline{3}}+\gamma:$

$$
\left\{\begin{array}{l}
\Sigma_{c}^{+} \rightarrow \Lambda_{c}^{+}+\gamma \\
\Xi_{c}^{\prime+} \rightarrow \Xi_{c}^{+}+\gamma \\
\Xi_{c}^{\prime 0} \rightarrow \Xi_{c}^{0}+\gamma
\end{array}\right.
$$

$B_{6}^{*} \rightarrow B_{6}+\gamma:$

$$
\left\{\begin{array}{l}
\Sigma_{c}^{*++} \rightarrow \Sigma_{c}^{++}+\gamma \\
\Sigma_{c}^{*+} \rightarrow \Sigma_{c}^{+}+\gamma \\
\Sigma_{c}^{* 0} \rightarrow \Sigma_{c}^{0}+\gamma \\
\Xi_{c}^{* \prime+} \rightarrow \Xi_{c}^{\prime+}+\gamma \\
\Xi_{c}^{* \prime} \rightarrow \Xi_{c}^{\prime 0}+\gamma \\
\Omega_{c}^{* 0} \rightarrow \Omega_{c}^{0}+\gamma
\end{array}\right.
$$




$$
B_{6}^{*} \rightarrow B_{\overline{3}}+\gamma:
$$

$$
\left\{\begin{array}{l}
\Sigma_{c}^{*+} \rightarrow \Lambda_{c}^{+}+\gamma \\
\Xi_{c}^{* \prime+} \rightarrow \Xi_{c}^{+}+\gamma \\
\Xi_{c}^{* \prime 0} \rightarrow \Xi_{c}^{0}+\gamma
\end{array}\right.
$$

The leading order Lagrangians in Eqs. (5)-(66) do not contribute to the above EM decays. So the lowest order EM decay diagrams arise from the $O\left(p^{2}\right)$ Lagrangians at the tree level. If we consider the next leading order corrections, we need the contribution from both the tree-level $O\left(p^{3}\right)$ Lagrangians and the chiral loop corrections from the leading order Lagrangians.

The higher ordered Lagrangian can be constructed with the help of the new building blocks $f_{\mu \nu}^{ \pm}$.

$$
\begin{aligned}
& f_{\mu \nu}^{ \pm}=u^{\dagger} f_{\mu \nu}^{R} u \pm u f_{\mu \nu}^{L} u^{\dagger} \\
& f_{\mu \nu}^{R}=\partial_{\mu} r_{\nu}-\partial_{\nu} r_{\mu}-i\left[r_{\mu}, r_{\nu}\right] \\
& f_{\mu \nu}^{L}=\partial_{\mu} l_{\nu}-\partial_{\nu} l_{\mu}-i\left[l_{\mu}, l_{\nu}\right]
\end{aligned}
$$

$r_{\mu}=l_{\mu}=-e Q_{B} \mathcal{A}_{\mu}, \quad Q_{B}=\left(\begin{array}{lll}2 & 0 & 0 \\ 0 & 0 & 0 \\ 0 & 0 & 0\end{array}\right)$ for the charmed baryons, $\quad Q_{B}=\left(\begin{array}{ccc}1 & 0 & 0 \\ 0 & -1 & 0 \\ 0 & 0 & -1\end{array}\right)$ for the bottomed baryons

The transformation property of the building blocks can be seen in Ref. 31]. What we need in the following is that the operator $f_{\mu \nu}^{ \pm}$transforms as the adjoint representation. We notice that the direct product of $3 \times 6=8+10$ contains only one adjoint representation. Therefore, there is one independent interaction term in the $O\left(p^{2}\right)$ Lagrangians for the EM transitions between the $\overline{3}$ and $6 / 6^{*}$-dimensional baryons.

In contrast, the direct product of $\overline{6} \times 6$ contains one singlet and one octet. Accordingly, there are two independent interaction terms for the EM transitions between the 6 and $6^{*}$-dimensional baryons. The octet piece corresponds to the $f_{\mu \nu}^{+}$term while the singlet piece corresponds to the trace of $f_{\mu \nu}^{+}$in Eq.(12). The $O\left(p^{2}\right)$ Lagrangians are as follows

$$
\left\{\begin{array}{l}
\mathcal{L}_{\overline{3} 6}^{(2)}=\frac{f_{2}}{\Lambda_{\chi}} \operatorname{Tr} \bar{B}_{\overline{3}} \sigma^{\mu \nu} f_{\mu \nu}^{+} B_{6}+\text { h.c. } \\
\mathcal{L}_{66^{*}}^{(2)}=\frac{f_{3}}{\Lambda_{\chi}^{2}} \operatorname{Tr} \bar{B}_{6} \epsilon^{\mu \nu \rho \sigma} f_{\mu \nu}^{+} i D_{\rho} B_{\sigma}^{6^{*}}+\frac{\tilde{f}_{3}}{\Lambda_{\chi}^{2}} \operatorname{Tr} \bar{B}_{6} \epsilon^{\mu \nu \rho \sigma}\left\langle f_{\mu \nu}^{+}\right\rangle i D_{\rho} B_{\sigma}^{6^{*}}+\text { h.c. } \\
\mathcal{L}_{\overline{3} 6^{*}}^{(2)}=\frac{f_{4}}{\Lambda_{\chi}^{2}} \operatorname{Tr} \bar{B}_{\overline{3}} \epsilon^{\mu \nu \rho \sigma} f_{\mu \nu}^{+} i D_{\rho} B_{\sigma}^{6^{*}}+\text { h.c. }
\end{array}\right.
$$

where $\operatorname{Tr}$ and \langle\rangle denote the trace in the flavor space, and $\Lambda_{\chi}=4 \pi F_{0}$. The coefficients $f_{i}$ 's are the new dimensionless LECs. The 8-dimensional operator $f_{\mu \nu}^{+}$and the $\overline{3}, 6$-dimensional baryon fields couple into the possible flavor singlet.

Similarly, the $O\left(p^{3}\right)$ Lagrangians for the EM decays are constructed as

$$
\begin{aligned}
\mathcal{L}_{66^{*}}^{(3)}= & \frac{h_{1}}{\Lambda_{\chi}^{3}} \operatorname{Tr} \bar{B}_{6} \nabla_{\lambda} f_{\mu \nu}^{+} \gamma^{\lambda} \gamma_{5} i D^{[\mu} B_{6^{*}}^{\nu]}+\frac{h_{2}}{\Lambda_{\chi}^{3}} \operatorname{Tr} \bar{B}_{6} \nabla_{\lambda} f_{\mu \nu}^{+} \gamma^{[\mu} \gamma_{5} i D^{\nu]} B_{6^{*}}^{\lambda}+\frac{h_{3}}{\Lambda_{\chi}^{3}} \operatorname{Tr} \bar{B}_{6} \nabla_{\lambda} f_{\mu \nu}^{+} i D^{\lambda} \gamma^{[\mu} \gamma_{5} B_{6^{*}}^{\nu]}+\text { h.c. } \\
& +\frac{\tilde{h}_{1}}{\Lambda_{\chi}^{3}} \operatorname{Tr} \bar{B}_{6} \nabla_{\lambda}\left\langle f_{\mu \nu}^{+}\right\rangle \gamma^{\lambda} \gamma_{5} i D^{[\mu} B_{6^{*}}^{\nu]}+\frac{\tilde{h}_{2}}{\Lambda_{\chi}^{3}} \operatorname{Tr} \bar{B}_{6} \nabla_{\lambda}\left\langle f_{\mu \nu}^{+}\right\rangle \gamma^{[\mu} \gamma_{5} i D^{\nu]} B_{6^{*}}^{\lambda}+\frac{\tilde{h}_{3}}{\Lambda_{\chi}^{3}} \operatorname{Tr} \bar{B}_{6} \nabla_{\lambda}\left\langle f_{\mu \nu}^{+}\right\rangle i D^{\lambda} \gamma^{[\mu} \gamma_{5} B_{6^{*}}^{\nu]}+\text { h.c. } \\
\mathcal{L}_{\overline{3} 6^{*}}^{(3)}= & \frac{h_{4}}{\Lambda_{\chi}^{3}} \operatorname{Tr} \bar{B}_{\overline{3}} \nabla_{\lambda} f_{\mu \nu}^{+} \gamma^{\lambda} \gamma_{5} i D^{[\mu} B_{6^{*}}^{\nu]}+\frac{h_{5}}{\Lambda_{\chi}^{3}} \operatorname{Tr} \bar{B}_{\overline{3}} \nabla_{\lambda} f_{\mu \nu}^{+} \gamma^{[\mu} \gamma_{5} i D^{\nu]} B_{6^{*}}^{\lambda}+\frac{h_{6}}{\Lambda_{\chi}^{3}} \operatorname{Tr} \bar{B}_{\overline{3}} \nabla_{\lambda} f_{\mu \nu}^{+} i D^{\lambda} \gamma^{[\mu} \gamma_{5} B_{6^{*}}^{\nu]}+\text { h.c. }
\end{aligned}
$$

where $D^{[\mu} B^{\nu]}=D^{\mu} B^{\nu}-D^{\nu} B^{\mu}$ and the other commutators are similar. The coefficients $h_{i}$ 's are also dimensionless LECs.

Because of the constraint of the above flavor structure, we notice that there does not exist EM transitions between the neutral $\overline{3}$ and $6 / 6^{*}$-dimensional baryons at the tree level up to $O\left(p^{3}\right)$. This seemingly surprising result is the manifestation of the famous flavor SU(3) U-spin symmetry, which interchanges the strange and down quark. Notice that the s and d quarks carry the same charge. With the exact SU(3) flavor symmetry, the s and d quarks are identical 
particles both in QCD and QED! Therefore, the Pauli principle forbids the s and d pair within the $\Xi_{c}^{0}$ baryon to form the flavor antitriplet. In other words, the decay processes $\Xi_{c}^{\prime 0} \rightarrow \Xi_{c}^{0}+\gamma$ and $\Xi_{c}^{* \prime 0} \rightarrow \Xi_{c}^{0}+\gamma$ are forbidden at the tree level up to $O\left(p^{3}\right)$.

If we consider the explicit SU(3) symmetry breaking, we can construct the tree-level Lagrangians at $O\left(p^{4}\right)$ such as

$$
\mathcal{L}^{(4)} \propto \operatorname{Tr} \bar{B}_{\overline{3}} \chi_{+} \sigma^{\mu \nu}\left\langle f_{\mu \nu}^{+}\right\rangle B_{6}+\text { h.c. }
$$

where $\chi_{+}$is the building block relevant to the current quark mass [31]. Since we truncate the chiral expansion at $O\left(p^{3}\right)$, we do not try to exhaust all the possible $O\left(p^{4}\right)$ Lagrangians.

On the other hand, the decay processes $\Xi_{c}^{\prime 0} \rightarrow \Xi_{c}^{0}+\gamma$ and $\Xi_{c}^{* \prime 0} \rightarrow \Xi_{c}^{0}+\gamma$ can occur through the chiral loops at $O\left(p^{3}\right)$ if we consider the explicit SU(3) symmetry breaking. Its divergence would be absorbed by the above tree-level Lagrangians at $O\left(p^{4}\right)$. We will include these corrections carefully in the following section.

\section{THE EM DECAY WIDTHS IN HBCHPT}

\section{A. The EM Decay Widths at $O\left(p^{2}\right)$}

We calculate the EM decay amplitude in Coulomb gauge. For a nonpolarized process, the summation of the photon polarizationyields the metric $h_{\mu \nu}$

$$
\sum_{r=1,2} \epsilon_{\mu}^{r *} \epsilon_{\nu}^{r}=-h_{\mu \nu}
$$

We adopt the metric $h_{\mu \nu}$ in Ref. [38].

$$
h_{\mu \nu}=g_{\mu \nu}-\frac{l \cdot n\left(l_{\mu} n_{\nu}+l_{\nu} n_{\mu}\right)-l_{\mu} l_{\nu}}{(l \cdot n)^{2}-l^{2}}
$$

where $n=(1,0,0,0)$ and $l$ is the photon 4-momentum. We plot the lowest order Feynman diagrams from Eq.(12) in Fig. 1. The single line represents a spin- $\frac{1}{2}$ baryon and double line a spin- $\frac{3}{2}$ baryon. The solid dot represents an $O\left(p^{2}\right)$
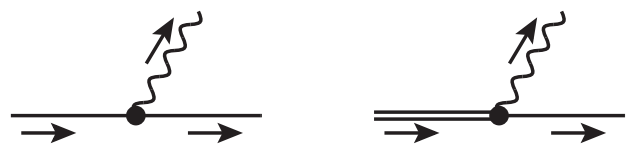

FIG. 1: The $O\left(p^{2}\right)$ tree level process

EM vertex.

Summing the photon polarization $r$, the spin directions of the initial and final baryon states $S_{A}$, $S_{B}$, we obtain the square of EM decay amplitude modular for the spin- $\frac{1}{2}$ to $\frac{1}{2}$ and spin- $\frac{3}{2}$ to $\frac{1}{2}$ transitions respectively.

$$
\begin{gathered}
\sum_{r, S_{A}, S_{B}}\left|\mathcal{M}_{\frac{1}{2}-\frac{1}{2}}^{(2)}\right|^{2}=16 e^{2}\left|D_{i j}^{(2)}\right|^{2} M_{A} M_{B} \ell^{2} \\
\sum_{r, S_{A}, S_{B}}\left|\mathcal{M}_{\frac{1}{2}-\frac{3}{2}}^{(2)}\right|^{2}=\frac{128}{3} e^{2}\left|D_{i j}^{(2)}\right|^{2} M_{A} M_{B}^{3} \ell^{2}
\end{gathered}
$$

where $M_{A}, M_{B}$ represent the baryon mass of the initial and the final states. $\ell$ is the modular of the photon 3momentum. In the rest frame of the initial state, $\ell$ only depends on the baryon mass.

$$
\ell=\frac{\left(M_{A}+M_{B}\right)\left(M_{B}-M_{A}\right)}{2 M_{B}}
$$

For each channel, the coefficients $D_{i j}^{(2)}$ 's are listed in the 2nd column of Table \.

Since some LECs always appear in a combined form in the decay amplitudes, we abbreviate $h_{i j}=h_{i}+h_{j}$ where $h_{i}$ etc arises from the tree-level Lagrangians at $O\left(p^{3}\right)$. We also collect the LECs $h_{i j}=h_{i}+h_{j}$ in Table I For the bottomed baryon systems, the LECs $f^{b}, h^{b}$ are marked with the superscript $b$ for distinction. The corresponding coefficients are collected in Table \. 
TABLE I: The coefficients $D_{i j}^{(2)}, D_{i j, 1}^{(3)}, D_{i j, 2}^{(3)}$ in the EM decay amplitudes.

\begin{tabular}{cccc}
\hline EM decay processes & Coeff. $D_{i j}^{(2)}$ of $O\left(p^{2}\right)$ & Coeff. $D_{i j, 1}^{(3)}$ of $O\left(p^{3}\right)$ & Coeff. $D_{i j, 2}^{(3)}$ of $O\left(p^{3}\right)$ \\
\hline$\Sigma_{c}^{+} \rightarrow \gamma+\Lambda_{c}^{+}$ & $-\frac{4 \sqrt{2} f_{2}}{\Lambda_{\chi}}$ & - & - \\
$\Xi_{c}^{\prime+} \rightarrow \gamma+\Xi_{c}^{+}$ & $-\frac{4 \sqrt{2} f_{2}}{\Lambda_{\chi}}$ & - & - \\
$\Xi_{c}^{\prime 0} \rightarrow \gamma+\Xi_{c}^{0}$ & - & - & - \\
\hline$\Sigma_{c}^{*++} \rightarrow \gamma+\Sigma_{c}^{++}$ & $-\frac{4\left(f_{3}+f_{3}\right)}{\Lambda_{\chi}^{2}}$ & $\frac{16\left(h_{13}+h_{13}\right)}{\Lambda_{\chi}^{3}}$ & $-\frac{16\left(h_{23}+h_{23}\right)}{\Lambda_{\chi}^{3}}$ \\
$\Sigma_{c}^{*+} \rightarrow \gamma+\Sigma_{c}^{+}$ & $-\frac{2\left(f_{3}+2 \tilde{f}_{3}\right)}{\Lambda_{\chi}^{2}}$ & $\frac{8\left(h_{13}+\tilde{h}_{13}\right)}{\Lambda_{\chi}^{3}}$ & $-\frac{16\left(h_{23}+\tilde{h}_{23}\right)}{\Lambda_{\chi}^{3}}$ \\
$\Sigma_{c}^{* 0} \rightarrow \gamma+\Sigma_{c}^{0}$ & $-\frac{4 \tilde{f}_{\chi}}{\Lambda_{\chi}^{2}}$ & $\frac{16 \tilde{h}_{13}}{\Lambda_{\chi}^{3}}$ & $-\frac{16 \tilde{h}_{23}}{\Lambda_{\chi}^{3}}$ \\
$\Xi_{c}^{* \prime+} \rightarrow \gamma+\Xi_{c}^{\prime+}$ & $-\frac{2\left(f_{3}+2 \tilde{f}_{3}\right)}{\Lambda_{\chi}^{2}}$ & $\frac{8\left(h_{13}+\tilde{h}_{13}\right)}{\Lambda_{\chi}^{3}}$ & $-\frac{16\left(h_{23}+\tilde{h}_{23}\right)}{\Lambda_{\chi}^{3}}$ \\
$\Xi_{c}^{* \prime 0} \rightarrow \gamma+\Xi_{c}^{\prime 0}$ & $-\frac{4 \tilde{f}_{\chi}}{\Lambda_{\chi}^{2}}$ & $\frac{16 \tilde{h}_{13}}{\Lambda_{\chi}^{3}}$ & $-\frac{16 \tilde{h}_{23}}{\Lambda_{\chi}^{3}}$ \\
$\Omega_{c}^{* 0} \rightarrow \gamma+\Omega_{c}^{0}$ & $-\frac{4 \tilde{f}_{2}}{\Lambda_{\chi}^{2}}$ & $\frac{16 \tilde{h}_{13}}{\Lambda_{\chi}^{3}}$ & $-\frac{16 \tilde{h}_{23}}{\Lambda_{\chi}^{3}}$ \\
\hline$\Sigma_{c}^{*+} \rightarrow \gamma+\Lambda_{c}^{+}$ & $-\frac{2 \sqrt{2} f_{4}}{\Lambda_{\chi}^{2}}$ & $\frac{8 \sqrt{2} h_{46}}{\Lambda_{\chi}^{3}}$ & $-\frac{8 \sqrt{2} h_{56}}{\Lambda_{\chi}^{3}}$ \\
$\Xi_{c}^{*+} \rightarrow \gamma+\Xi_{c}^{+}$ & $-\frac{2 \sqrt{2} f_{4}}{\Lambda_{\chi}^{2}}$ & $\frac{8 \sqrt{2} h_{46}}{\Lambda_{\chi}^{3}}$ & $-\frac{8 \sqrt{2} h_{56}}{\Lambda_{\chi}^{3}}$ \\
$\Xi_{c}^{* \prime 0} \rightarrow \gamma+\Xi_{c}^{0}$ & - & - & - \\
\hline
\end{tabular}

TABLE II: The coefficients $D_{i j}^{(2)}, D_{i j, 1}^{(3)}, D_{i j, 2}^{(3)}$ for the bottomed baryons.

\begin{tabular}{cccc}
\hline EM decay processes & Coeff. $D_{i j}^{(2)}$ of $O\left(p^{2}\right)$ & Coeff. $D_{i j, 1}^{(3)}$ of $O\left(p^{3}\right)$ & Coeff. $D_{i j, 2}^{(3)}$ of $O\left(p^{3}\right)$ \\
\hline$\Sigma_{b}^{0} \rightarrow \gamma+\Lambda_{b}^{0}$ & $-\frac{4 \sqrt{2} f_{2}^{b}}{\Lambda_{\chi}}$ & - & - \\
$\Xi_{b}^{\prime 0} \rightarrow \gamma+\Xi_{b}^{0}$ & $-\frac{4 \sqrt{2} f_{2}^{b}}{\Lambda_{\chi}}$ & - & - \\
$\Xi_{b}^{\prime-} \rightarrow \gamma+\Xi_{b}^{-}$ & - & - & - \\
\hline$\Sigma_{b}^{*+} \rightarrow \gamma+\Sigma_{b}^{+}$ & $\frac{2\left(-f_{3}^{b}+\tilde{f}_{3}^{b}\right)}{\Lambda_{\chi}^{2}}$ & $-\frac{8\left(-h_{13}^{b}+\tilde{h}_{13}^{b}\right)}{\Lambda_{\chi}^{3}}$ & $\frac{8\left(-h_{23}^{b}+\tilde{h}_{23}^{b}\right)}{\Lambda_{\chi}^{3}}$ \\
$\Sigma_{b}^{* 0} \rightarrow \gamma+\Sigma_{b}^{0}$ & $\frac{2 \tilde{f}_{3}^{b}}{\Lambda_{\chi}^{2}}$ & $-\frac{8 \tilde{h}_{13}^{b}}{\Lambda_{\chi}^{3}}$ & $\frac{8 \tilde{h}_{23}^{b}}{\Lambda_{\chi}^{3}}$ \\
$\Sigma_{b}^{*-} \rightarrow \gamma+\Sigma_{b}^{-}$ & $\frac{2\left(f_{3}^{b}+\tilde{f}_{3}^{b}\right)}{\Lambda_{\chi}^{2}}$ & $-\frac{8\left(h_{13}^{b}+\tilde{h}_{13}^{b}\right)}{\Lambda_{\chi}^{3}}$ & $\frac{8\left(h_{23}^{b}+\tilde{h}_{23}^{b}\right)}{\Lambda_{\chi}^{3}}$ \\
$\Xi_{b}^{* \prime 0} \rightarrow \gamma+\Xi_{b}^{\prime 0}$ & $\frac{2 f_{3}^{b}}{\Lambda_{\chi}^{2}}$ & $-\frac{8 \tilde{h}_{13}^{b}}{\Lambda_{\chi}^{3}}$ & $\frac{8 \tilde{h}_{23}^{b}}{\Lambda_{\chi}^{3}}$ \\
$\Xi_{b}^{* \prime-} \rightarrow \gamma+\Xi_{b}^{\prime-}$ & $\frac{2\left(f_{3}^{b}+\tilde{f}_{3}^{b}\right)}{\Lambda_{\chi}^{2}}$ & $-\frac{8\left(h_{13}^{b}+\tilde{h}_{13}^{b}\right)}{\Lambda_{\chi}^{3}}$ & $\frac{8\left(h_{13}+\tilde{h}_{13}\right)}{\Lambda_{\chi}^{3}}$ \\
$\Omega_{b}^{*-} \rightarrow \gamma+\Omega_{b}^{-}$ & $\frac{2\left(f_{3}^{b}+\tilde{f}_{3}^{b}\right)}{\Lambda_{\chi}^{2}}$ & $-\frac{8\left(h_{13}^{b}+\tilde{h}_{13}^{b}\right)}{\Lambda_{\chi}^{3}}$ & $\frac{8\left(h_{13}^{b}+\tilde{h}_{13}^{b}\right)}{\Lambda_{\chi}^{3}}$ \\
\hline$\Sigma_{b}^{* 0} \rightarrow \gamma+\Lambda_{b}^{0}$ & $-\frac{2 \sqrt{2} f_{4}^{b}}{\Lambda_{\chi}^{2}}$ & $\frac{8 \sqrt{2} \hat{h}_{46}^{b}}{\Lambda_{\chi}^{3}}$ & $-\frac{8 \sqrt{2} \tilde{h}_{56}^{b}}{\Lambda_{\chi}^{3}}$ \\
$\Xi_{b}^{* \prime 0} \rightarrow \gamma+\Xi_{b}^{0}$ & $-\frac{2 \sqrt{2} f_{4}^{b}}{\Lambda_{\chi}^{2}}$ & $\frac{8 \sqrt{2} \tilde{h}_{46}^{b}}{\Lambda_{\chi}^{3}}$ & $-\frac{8 \sqrt{2} \tilde{h}_{56}^{b}}{\Lambda_{\chi}^{3}}$ \\
$\Xi_{b}^{* \prime-} \rightarrow \gamma+\Xi_{b}^{-}$ & - & - & - \\
\hline
\end{tabular}

\section{B. The $O\left(p^{3}\right)$ contributions at the tree level}

The next leading order contributions arise from the tree-level Lagrangians in Eqs. 1314) and chiral loop corrections from Eqs.(4)-(6). The Lagrangians in Eqs.(1213) contribute to the tree-level process between the spin- $\frac{1}{2}$ and $\frac{3}{2}$ baryons in Fig. 2 In fact, there are two other Lagrangians involving $f_{\mu \nu}^{+}$at $O\left(p^{3}\right)$. One is $\bar{B} \gamma^{\mu} \nabla^{\nu} f_{\mu \nu}^{+} B$ which leads to the EM decay amplitude with the factor $l_{\mu} l^{\nu} \epsilon_{\nu}-l^{2} \epsilon_{\mu}$. Under the Coulomb gauge, this term vanishes. The other possible interaction Lagrangian is $\bar{B} \gamma_{5} f_{\mu \nu}^{+} D_{\rho} B_{\sigma}$. In the leading order of the heavy baryon expansion, its contribution also vanishes since $\overline{\mathcal{N}} \gamma_{5} \mathcal{T}_{\sigma}=0$.

There are two Lorentz structures from Fig. 2. To sum up, all the EM decay amplitudes at the tree level up to $O\left(p^{3}\right)$ can be written as

$$
\left\{\begin{array}{l}
i \mathcal{M}^{(2)}=D^{(2)} M_{B}\left(l_{\mu} \epsilon_{\nu}-l_{\nu} \epsilon_{\mu}\right) \epsilon^{\mu \nu \rho \sigma} v_{\rho} \bar{u}^{S_{A}} u_{\sigma}^{S_{B}} \\
i \mathcal{M}^{(3)}=i D_{1}^{(3)} M_{B} v \cdot l \bar{u}^{S_{A}} S^{\mu^{\prime}} l_{\mu^{\prime}} \epsilon_{\sigma^{\prime}} u_{\sigma^{\prime}}^{S_{B}}+i D_{2}^{(3)} M_{B} v \cdot l \bar{u}^{S_{A}} S^{\mu^{\prime}} \epsilon_{\mu^{\prime}} l_{\sigma^{\prime}} u_{\sigma^{\prime}}^{S_{B}}
\end{array}\right.
$$

where the Pauli-Lubanski spin operator $S^{\mu}$ is defined as $S^{\mu}=-\frac{1}{2} \gamma_{5}\left(\gamma^{\mu} \psi-v^{\mu}\right)$. The coefficients $D_{1}^{(3)}, D_{2}^{(3)}$ 's are also 


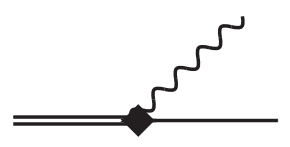

FIG. 2: The $O\left(p^{3}\right)$ tree level process.

listed in Table In Coulomb gauge, the cross term between the $O\left(p^{2}\right)$ and $O\left(p^{3}\right)$ EM decay amplitudes reads

$$
\sum_{r, S_{A}, S_{B}}\left(i \mathcal{M}^{(2)}\right)^{*} i \mathcal{M}^{(3)}+\left(i \mathcal{M}^{(3)}\right)^{*} i \mathcal{M}^{(2)}=-\frac{32}{3} M_{A} M_{B}^{3} \ell^{3} D^{(2)}\left(D_{1}^{(3)}-D_{2}^{(3)}\right)
$$

The square of the decay amplitude from the $O\left(p^{3}\right)$ diagram is

$$
\sum_{r, S_{A}, S_{B}}\left(\left|i \mathcal{M}_{1}\right|^{2}+\left|i \mathcal{M}_{2}\right|^{2}\right)=\frac{8}{3}\left(\left|D_{i j, 1}^{(3)}\right|^{2}+\left|D_{i j, 2}^{(3)}\right|^{2}\right) M_{A} M_{B}^{3} \ell^{4}
$$

\section{The $O\left(p^{3}\right)$ chiral loop contributions}

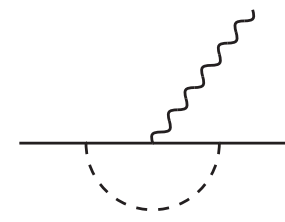

$a$

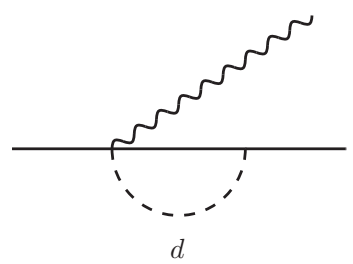

d

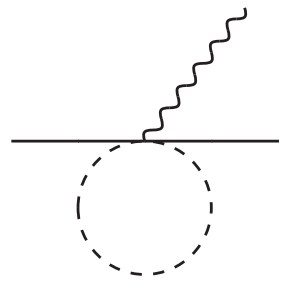

$b$

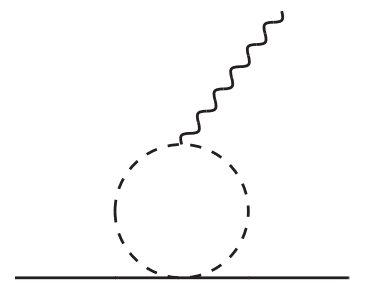

$e$

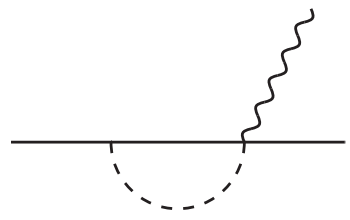

$c$

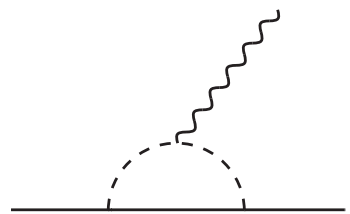

$f$

FIG. 3: $O\left(p^{3}\right)$ loop level process

At the one-loop level, there are six possible Feynman diagrams as shown in Fig. 3, All the vertices are from Eqs. (4516). In diagram a, the photon vertex is from the tree-level EM interaction term while the meson vertex from the strong interaction terms. In diagram $b$, the photon-meson vertex is from the chiral connection. In diagram $\mathrm{c}$ and $\mathrm{d}$, both two vertices are from the strong interaction and seagull terms. In diagram e, the meson vertex arises from the chiral connection.

The external baryons in diagrams b and e must be the same. So they do not contribute to the EM decay process. Diagrams c and d vanish in the heavy baryon mass limit. The nontrivial recoil corrections appear at $O\left(p^{4}\right)$ which is beyond our chiral truncation order in this work. The decay amplitude from diagram a between spin $\frac{3}{2}$ to $\frac{1}{2}$ baryons is proportional to either $S \cdot v=0$ or $v \cdot \mathcal{T}=0$. Hence its contribution vanishes. Similarly the decay amplitude between spin $\frac{1}{2}$ to $\frac{1}{2}$ baryons from diagram a is always proportional to $\epsilon \cdot v$. Under Coulomb gauge, this amplitude also vanishes.

Diagram $\mathrm{f}$ can be classified into two different types according to the Lorentz structure in the loop integrals, which are displayed in Fig. (4 For $g_{6}=0$, the contributions from two of these Feynman diagrams in Fig. 4 vanish. 


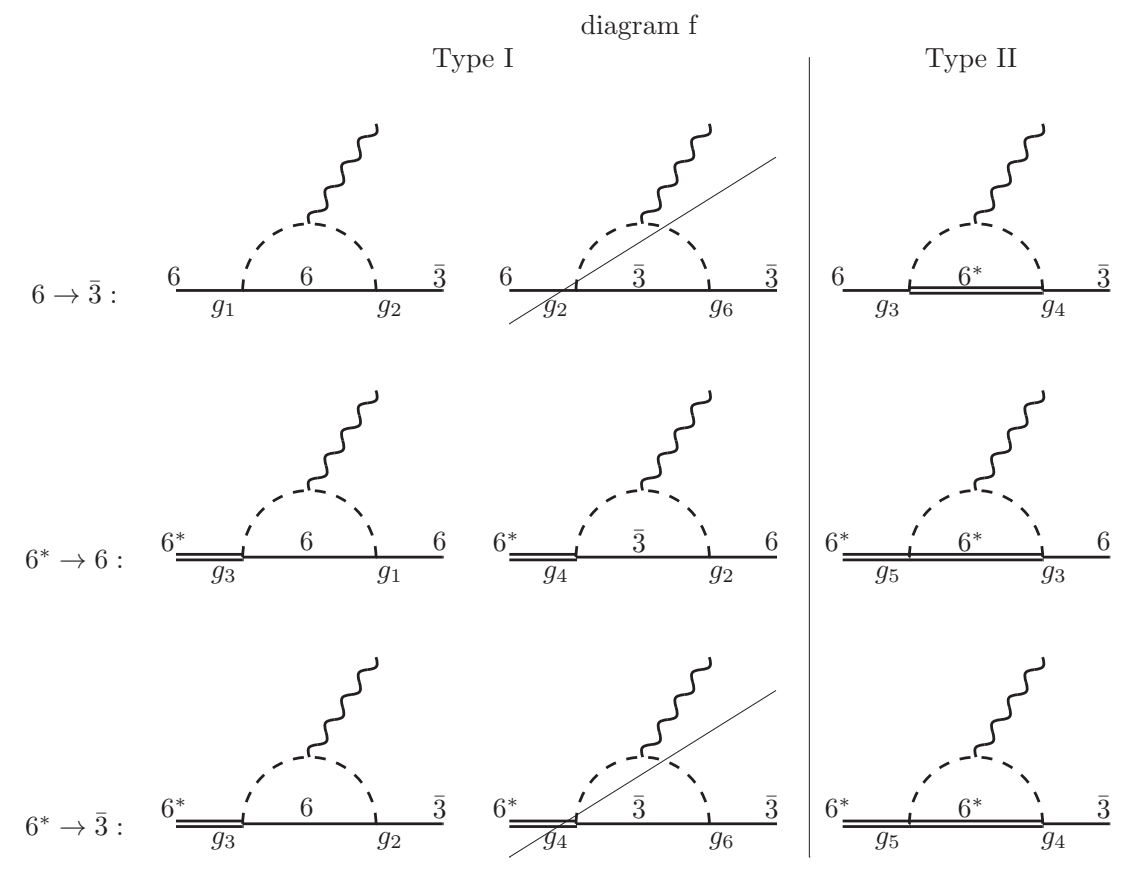

FIG. 4: The flavor structures of loop-f.

\section{1. $\operatorname{spin}-\frac{1}{2}$ to $\frac{1}{2}$ diagram}

For the $6 \rightarrow \overline{3} \mathrm{EM}$ decays, the decay amplitude from the chiral loops can be written as

$$
i \mathcal{M}^{\text {loop-f }}=i \mathcal{M}_{\mathrm{I}}+i \mathcal{M}_{\mathrm{II}}=\epsilon^{\mu \nu \alpha \beta} v_{\alpha} \bar{u}_{i}^{s_{A}} S_{\beta} u_{j}^{s_{B}}\left(l_{\mu} \epsilon_{\nu}-l_{\nu} \epsilon_{\mu}\right)\left[\frac{i}{2} b_{\mathrm{I}}-\frac{2}{3} i b_{\mathrm{II}}\right]
$$

The function $\left[\frac{i}{2} b_{\mathrm{I}}-\frac{2}{3} i b_{\mathrm{II}}\right]$ contains all the coefficients involving $g_{i}$ etc and the loop integrals. The loop-f Lorentz structure is the same as that of the $O\left(p^{2}\right)$ tree diagram. The divergence of the chiral loops can be absorbed by the $O\left(p^{2}\right)$ LECs. We list the functions of the three channels below.

$$
\begin{aligned}
\frac{i}{2} b_{\mathrm{I}}-\left.\frac{2}{3} i b_{\mathrm{II}}\right|_{\Lambda_{c}^{+} \leftarrow \Sigma_{c}^{+}}= & g_{3} g_{4} e\left(\frac{\gamma_{3}\left(m_{K},-\delta_{2}, \ell\right)}{6 \sqrt{2} F_{0}^{2}}+\frac{\gamma_{9}\left(m_{K},-\delta_{2}, \ell\right)}{3 \sqrt{2} F_{0}^{2}}+\frac{\sqrt{2} \gamma_{3}\left(m_{\pi},-\delta_{2}, \ell\right)}{3 F_{0}^{2}}+\frac{2 \sqrt{2} \gamma_{9}\left(m_{\pi},-\delta_{2}, \ell\right)}{3 F_{0}^{2}}\right) \\
& +g_{1} g_{2} e\left(\frac{\gamma_{3}\left(m_{K}, 0, \ell\right)}{2 \sqrt{2} F_{0}^{2}}+\frac{\gamma_{9}\left(m_{K}, 0, \ell\right)}{\sqrt{2} F_{0}^{2}}+\frac{\sqrt{2} \gamma_{3}\left(m_{\pi}, 0, \ell\right)}{F_{0}^{2}}+\frac{2 \sqrt{2} \gamma_{9}\left(m_{\pi}, 0, \ell\right)}{F_{0}^{2}}\right) \\
\frac{i}{2} b_{\mathrm{I}}-\left.\frac{2}{3} i b_{\mathrm{II}}\right|_{\Xi_{c}^{+} \leftarrow \Xi_{c}^{\prime+}}= & g_{3} g_{4} e\left(\frac{\gamma_{3}\left(m_{K},-\delta_{2}, \ell\right)}{6 \sqrt{2} F_{0}^{2}}+\frac{\gamma_{9}\left(m_{K},-\delta_{2}, \ell\right)}{3 \sqrt{2} F_{0}^{2}}+\frac{\sqrt{2} \gamma_{3}\left(m_{\pi},-\delta_{2}, \ell\right)}{3 F_{0}^{2}}+\frac{2 \sqrt{2} \gamma_{9}\left(m_{\pi},-\delta_{2}, \ell\right)}{3 F_{0}^{2}}\right) \\
& +g_{1} g_{2} e\left(\frac{\gamma_{3}\left(m_{K}, 0, \ell\right)}{2 \sqrt{2} F_{0}^{2}}+\frac{\gamma_{9}\left(m_{K}, 0, \ell\right)}{\sqrt{2} F_{0}^{2}}+\frac{\sqrt{2} \gamma_{3}\left(m_{\pi}, 0, \ell\right)}{F_{0}^{2}}+\frac{2 \sqrt{2} \gamma_{9}\left(m_{\pi}, 0, \ell\right)}{F_{0}^{2}}\right) \\
= & g_{3} g_{4} e\left(\frac{\gamma_{3}\left(m_{K},-\delta_{2}, \ell\right)}{6 \sqrt{2} F_{0}^{2}}+\frac{\gamma_{9}\left(m_{K},-\delta_{2}, \ell\right)}{3 \sqrt{2} F_{0}^{2}}-\frac{\gamma_{3}\left(m_{\pi},-\delta_{2}, \ell\right)}{6 \sqrt{2} F_{0}^{2}}-\frac{\gamma_{9}\left(m_{\pi},-\delta_{2}, \ell\right)}{3 \sqrt{2} F_{0}^{2}}\right) \\
\frac{i}{2} b_{\mathrm{I}}-\left.\frac{2}{3} i b_{\mathrm{II}}\right|_{\Xi_{c}^{0} \leftarrow \Xi_{c}^{\prime 0}} & +g_{1} g_{2} e\left(\frac{\gamma_{3}\left(m_{K}, 0, \ell\right)}{2 \sqrt{2} F_{0}^{2}}+\frac{\gamma_{9}\left(m_{K}, 0, \ell\right)}{\sqrt{2} F_{0}^{2}}-\frac{\gamma_{3}\left(m_{\pi}, 0, \ell\right)}{2 \sqrt{2} F_{0}^{2}}-\frac{\gamma_{9}\left(m_{\pi}, 0, \ell\right)}{\sqrt{2} F_{0}^{2}}\right)
\end{aligned}
$$

The baryon mass splitting and the meson type in the loop can be read from the functions directly. The function $\gamma_{i}(m, \delta, \ell)$ arises from the loop integral, which are defined in Appendix VII. 


\section{2. $\operatorname{spin}-\frac{3}{2}$ to $\frac{1}{2}$ diagram}

For the spin- $\frac{3}{2}$ to $\frac{1}{2}$ EM decays, the Lorentz structure of the loop-f amplitude is different from the structure of the $O\left(p^{2}\right)$ tree level one, but is the same as that of the $O\left(p^{3}\right)$ tree level one. Both type-I and II loop-f amplitudes have two structures, which are marked with the subscript 1,2 .

$$
\begin{aligned}
i \mathcal{M}_{1}^{\text {loop-f }} & =i \mathcal{M}_{\mathrm{I} 1}+i \mathcal{M}_{\mathrm{II} 1}=\bar{u}^{s_{A}} S \cdot l \epsilon \cdot u^{s_{B}}\left[2 b_{\mathrm{I} 1}+2 b_{\mathrm{II} 1}\right] \\
i \mathcal{M}_{2}^{\text {loop-f }} & =i \mathcal{M}_{\mathrm{I} 2}+i \mathcal{M}_{\mathrm{II} 2}=\bar{u}^{s_{A}} S \cdot \epsilon l \cdot u^{s_{B}}\left[2 b_{\mathrm{I} 2}+2 b_{\mathrm{II} 2}\right]
\end{aligned}
$$

The function $\left[2 b_{\mathrm{I}}+2 b_{\mathrm{II}}\right]$ contains all the coefficients involving the coupling constants and the loop integrals, too. For the six EM decays between the 6 and $6^{*}$-dimensional baryons, we have

$$
\begin{aligned}
& 2 b_{\mathrm{I} 1}+\left.2 b_{\mathrm{II} 1}\right|_{\Sigma_{c}^{++} \leftarrow \Sigma_{c}^{*++}}=g_{1} g_{3} e\left(-\frac{i \gamma_{3}\left(m_{K}, \delta_{2}, \ell\right)}{F_{0}^{2}}-\frac{i \gamma_{9}\left(m_{K}, \delta_{2}, \ell\right)}{F_{0}^{2}}-\frac{i \gamma_{3}\left(m_{\pi}, \delta_{2}, \ell\right)}{F_{0}^{2}}-\frac{i \gamma_{9}\left(m_{\pi}, \delta_{2}, \ell\right)}{F_{0}^{2}}\right) \\
& +g_{2} g_{4} e\left(-\frac{2 i \gamma_{3}\left(m_{K}, \delta_{3}, \ell\right)}{F_{0}^{2}}-\frac{2 i \gamma_{9}\left(m_{K}, \delta_{3}, \ell\right)}{F_{0}^{2}}-\frac{2 i \gamma_{3}\left(m_{\pi}, \delta_{3}, \ell\right)}{F_{0}^{2}}-\frac{2 i \gamma_{9}\left(m_{\pi}, \delta_{3}, \ell\right)}{F_{0}^{2}}\right) \\
& +g_{3} g_{5} e\left(\frac{2 i \gamma_{3}\left(m_{K}, 0, \ell\right)}{3 F_{0}^{2}}-\frac{i \gamma_{8}\left(m_{K}, 0, \ell\right)}{3 F_{0}^{2}}+\frac{2 i \gamma_{3}\left(m_{\pi}, 0, \ell\right)}{3 F_{0}^{2}}-\frac{i \gamma_{8}\left(m_{\pi}, 0, \ell\right)}{3 F_{0}^{2}}\right) \\
& 2 b_{\mathrm{I} 2}+\left.2 b_{\mathrm{II} 2}\right|_{\Sigma_{c}^{++} \leftarrow \Sigma_{c}^{*++}}=g_{1} g_{3} e\left(-\frac{i \gamma_{8}\left(m_{K}, \delta_{2}, \ell\right)}{F_{0}^{2}}-\frac{i \gamma_{8}\left(m_{\pi}, \delta_{2}, \ell\right)}{F_{0}^{2}}\right)+g_{2} g_{4} e\left(-\frac{2 i \gamma_{8}\left(m_{K}, \delta_{3}, \ell\right)}{F_{0}^{2}}-\frac{2 i \gamma_{8}\left(m_{\pi}, \delta_{3}, \ell\right)}{F_{0}^{2}}\right) \\
& +g_{3} g_{5} e\left(-\frac{i \gamma_{3}\left(m_{K}, 0, \ell\right)}{F_{0}^{2}}-\frac{i \gamma_{8}\left(m_{K}, 0, \ell\right)}{3 F_{0}^{2}}-\frac{i \gamma_{3}\left(m_{\pi}, 0, \ell\right)}{F_{0}^{2}}-\frac{i \gamma_{8}\left(m_{\pi}, 0, \ell\right)}{3 F_{0}^{2}}\right) \\
& 2 b_{\mathrm{I} 1}+\left.2 b_{\mathrm{II} 1}\right|_{\Sigma_{c}^{+} \leftarrow \Sigma_{c}^{*+}}=g_{1} g_{3} e\left(-\frac{i \gamma_{3}\left(m_{K}, \delta_{2}, \ell\right)}{2 F_{0}^{2}}-\frac{i \gamma_{9}\left(m_{K}, \delta_{2}, \ell\right)}{2 F_{0}^{2}}\right)+g_{2} g_{4} e\left(-\frac{i \gamma_{3}\left(m_{K}, \delta_{3}, \ell\right)}{F_{0}^{2}}-\frac{i \gamma_{9}\left(m_{K}, \delta_{3}, \ell\right)}{F_{0}^{2}}\right) \\
& +g_{3} g_{5} e\left(\frac{i \gamma_{3}\left(m_{K}, 0, \ell\right)}{3 F_{0}^{2}}-\frac{i \gamma_{8}\left(m_{K}, 0, \ell\right)}{6 F_{0}^{2}}\right) \\
& 2 b_{\mathrm{I} 2}+\left.2 b_{\mathrm{II} 2}\right|_{\Sigma_{c}^{+} \leftarrow \Sigma_{c}^{*+}}=-\frac{i g_{1} g_{3} e \gamma_{8}\left(m_{K}, \delta_{2}, \ell\right)}{2 F_{0}^{2}}-\frac{i g_{2} g_{4} e \gamma_{8}\left(m_{K}, \delta_{3}, \ell\right)}{F_{0}^{2}}+g_{3} g_{5} e\left(-\frac{i \gamma_{3}\left(m_{K}, 0, \ell\right)}{2 F_{0}^{2}}-\frac{i \gamma_{8}\left(m_{K}, 0, \ell\right)}{6 F_{0}^{2}}\right) \\
& 2 b_{\mathrm{I} 1}+\left.2 b_{\mathrm{II} 1}\right|_{\Sigma_{c}^{0} \leftarrow \Sigma_{c}^{* 0}}=g_{1} g_{3} e\left(\frac{i \gamma_{3}\left(m_{\pi}, \delta_{2}, \ell\right)}{F_{0}^{2}}+\frac{i \gamma_{9}\left(m_{\pi}, \delta_{2}, \ell\right)}{F_{0}^{2}}\right)+g_{2} g_{4} e\left(\frac{2 i \gamma_{3}\left(m_{\pi}, \delta_{3}, \ell\right)}{F_{0}^{2}}+\frac{2 i \gamma_{9}\left(m_{\pi}, \delta_{3}, \ell\right)}{F_{0}^{2}}\right) \\
& +g_{3} g_{5} e\left(\frac{i \gamma_{8}\left(m_{\pi}, 0, \ell\right)}{3 F_{0}^{2}}-\frac{2 i \gamma_{3}\left(m_{\pi}, 0, \ell\right)}{3 F_{0}^{2}}\right) \\
& 2 b_{\mathrm{I} 2}+\left.2 b_{\mathrm{II} 2}\right|_{\Sigma_{c}^{0} \leftarrow \Sigma_{c}^{* 0}}=\frac{i g_{1} g_{3} e \gamma_{8}\left(m_{\pi}, \delta_{2}, \ell\right)}{F_{0}^{2}}+\frac{2 i g_{2} g_{4} e \gamma_{8}\left(m_{\pi}, \delta_{3}, \ell\right)}{F_{0}^{2}}+g_{3} g_{5} e\left(\frac{i \gamma_{3}\left(m_{\pi}, 0, \ell\right)}{F_{0}^{2}}+\frac{i \gamma_{8}\left(m_{\pi}, 0, \ell\right)}{3 F_{0}^{2}}\right) \\
& 2 b_{\mathrm{I} 1}+\left.2 b_{\mathrm{II} 1}\right|_{\Xi_{c}^{\prime+} \leftarrow \Xi_{c}^{* \prime+}}=g_{1} g_{3} e\left(-\frac{i \gamma_{3}\left(m_{\pi}, \delta_{2}, \ell\right)}{2 F_{0}^{2}}-\frac{i \gamma_{9}\left(m_{\pi}, \delta_{2}, \ell\right)}{2 F_{0}^{2}}\right)+g_{2} g_{4} e\left(-\frac{i \gamma_{3}\left(m_{\pi}, \delta_{3}, \ell\right)}{F_{0}^{2}}-\frac{i \gamma_{9}\left(m_{\pi}, \delta_{3}, \ell\right)}{F_{0}^{2}}\right) \\
& +g_{3} g_{5} e\left(\frac{i \gamma_{3}\left(m_{\pi}, 0, \ell\right)}{3 F_{0}^{2}}-\frac{i \gamma_{8}\left(m_{\pi}, 0, \ell\right)}{6 F_{0}^{2}}\right) \\
& 2 b_{\mathrm{I} 2}+\left.2 b_{\mathrm{II} 2}\right|_{\Xi_{c}^{\prime+} \leftarrow \Xi_{c}^{* \prime}}=-\frac{i g_{1} g_{3} e \gamma_{8}\left(m_{\pi}, \delta_{2}, \ell\right)}{2 F_{0}^{2}}-\frac{i g_{2} g_{4} e \gamma_{8}\left(m_{\pi}, \delta_{3}, \ell\right)}{F_{0}^{2}}+g_{3} g_{5} e\left(-\frac{i \gamma_{3}\left(m_{\pi}, 0, \ell\right)}{2 F_{0}^{2}}-\frac{i \gamma_{8}\left(m_{\pi}, 0, \ell\right)}{6 F_{0}^{2}}\right) \\
& 2 b_{\mathrm{I} 1}+\left.2 b_{\mathrm{II} 1}\right|_{\Xi_{c}^{\prime 0} \leftarrow \Xi_{c}^{* \prime 0}}=g_{1} g_{3} e\left(\frac{i \gamma_{3}\left(m_{K}, \delta_{2}, \ell\right)}{2 F_{0}^{2}}+\frac{i \gamma_{9}\left(m_{K}, \delta_{2}, \ell\right)}{2 F_{0}^{2}}+\frac{i \gamma_{3}\left(m_{\pi}, \delta_{2}, \ell\right)}{2 F_{0}^{2}}+\frac{i \gamma_{9}\left(m_{\pi}, \delta_{2}, \ell\right)}{2 F_{0}^{2}}\right) \\
& +g_{2} g_{4} e\left(\frac{i \gamma_{3}\left(m_{K}, \delta_{3}, \ell\right)}{F_{0}^{2}}+\frac{i \gamma_{9}\left(m_{K}, \delta_{3}, \ell\right)}{F_{0}^{2}}+\frac{i \gamma_{3}\left(m_{\pi}, \delta_{3}, \ell\right)}{F_{0}^{2}}+\frac{i \gamma_{9}\left(m_{\pi}, \delta_{3}, \ell\right)}{F_{0}^{2}}\right) \\
& +g_{3} g_{5} e\left(-\frac{i \gamma_{3}\left(m_{K}, 0, \ell\right)}{3 F_{0}^{2}}+\frac{i \gamma_{8}\left(m_{K}, 0, \ell\right)}{6 F_{0}^{2}}-\frac{i \gamma_{3}\left(m_{\pi}, 0, \ell\right)}{3 F_{0}^{2}}+\frac{i \gamma_{8}\left(m_{\pi}, 0, \ell\right)}{6 F_{0}^{2}}\right) \\
& 2 b_{\mathrm{I} 2}+\left.2 b_{\mathrm{II} 2}\right|_{\Xi_{c}^{\prime 0} \leftarrow \Xi_{c}^{* \prime 0}}=g_{1} g_{3} e\left(\frac{i \gamma_{8}\left(m_{K}, \delta_{2}, \ell\right)}{2 F_{0}^{2}}+\frac{i \gamma_{8}\left(m_{\pi}, \delta_{2}, \ell\right)}{2 F_{0}^{2}}\right)+g_{2} g_{4} e\left(\frac{i \gamma_{8}\left(m_{K}, \delta_{3}, \ell\right)}{F_{0}^{2}}+\frac{i \gamma_{8}\left(m_{\pi}, \delta_{3}, \ell\right)}{F_{0}^{2}}\right) \\
& +g_{3} g_{5} e\left(\frac{i \gamma_{3}\left(m_{K}, 0, \ell\right)}{2 F_{0}^{2}}+\frac{i \gamma_{8}\left(m_{K}, 0, \ell\right)}{6 F_{0}^{2}}+\frac{i \gamma_{3}\left(m_{\pi}, 0, \ell\right)}{2 F_{0}^{2}}+\frac{i \gamma_{8}\left(m_{\pi}, 0, \ell\right)}{6 F_{0}^{2}}\right)
\end{aligned}
$$




$$
\begin{aligned}
2 b_{\mathrm{I} 1}+\left.2 b_{\mathrm{II} 1}\right|_{\Omega_{c}^{0} \leftarrow \Omega_{c}^{* 0}}= & g_{1} g_{3} e\left(\frac{i \gamma_{3}\left(m_{K}, \delta_{2}, \ell\right)}{F_{0}^{2}}+\frac{i \gamma_{9}\left(m_{K}, \delta_{2}, \ell\right)}{F_{0}^{2}}\right)+g_{2} g_{4} e\left(\frac{2 i \gamma_{3}\left(m_{K}, \delta_{3}, \ell\right)}{F_{0}^{2}}+\frac{2 i \gamma_{9}\left(m_{K}, \delta_{3}, \ell\right)}{F_{0}^{2}}\right) \\
& +g_{3} g_{5} e\left(\frac{i \gamma_{8}\left(m_{K}, 0, \ell\right)}{3 F_{0}^{2}}-\frac{2 i \gamma_{3}\left(m_{K}, 0, \ell\right)}{3 F_{0}^{2}}\right) \\
2 b_{\mathrm{I} 2}+\left.2 b_{\mathrm{II} 2}\right|_{\Omega_{c}^{0} \leftarrow \Omega_{c}^{* 0}}= & \frac{i g_{1} g_{3} e \gamma_{8}\left(m_{K}, \delta_{2}, \ell\right)}{F_{0}^{2}}+\frac{2 i g_{2} g_{4} e \gamma_{8}\left(m_{K}, \delta_{3}, \ell\right)}{F_{0}^{2}}+g_{3} g_{5} e\left(\frac{i \gamma_{3}\left(m_{K}, 0, \ell\right)}{F_{0}^{2}}+\frac{i \gamma_{8}\left(m_{K}, 0, \ell\right)}{3 F_{0}^{2}}\right)
\end{aligned}
$$

For the three EM decays between the $\overline{3}$ and $6^{*}$-dimensional baryons, we have

$$
\begin{aligned}
& 2 b_{\mathrm{I} 1}+\left.2 b_{\mathrm{II} 1}\right|_{\Lambda_{c}^{+} \leftarrow \Sigma_{c}^{*+}}=g_{2} g_{3} e\left(-\frac{i \gamma_{3}\left(m_{K}, \delta_{2}, \ell\right)}{\sqrt{2} F_{0}^{2}}-\frac{i \gamma_{9}\left(m_{K}, \delta_{2}, \ell\right)}{\sqrt{2} F_{0}^{2}}-\frac{2 i \sqrt{2} \gamma_{3}\left(m_{\pi}, \delta_{2}, \ell\right)}{F_{0}^{2}}-\frac{2 i \sqrt{2} \gamma_{9}\left(m_{\pi}, \delta_{2}, \ell\right)}{F_{0}^{2}}\right) \\
& +g_{4} g_{5} e\left(\frac{i \sqrt{2} \gamma_{3}\left(m_{K}, 0, \ell\right)}{3 F_{0}^{2}}-\frac{i \gamma_{8}\left(m_{K}, 0, \ell\right)}{3 \sqrt{2} F_{0}^{2}}+\frac{4 i \sqrt{2} \gamma_{3}\left(m_{\pi}, 0, \ell\right)}{3 F_{0}^{2}}-\frac{2 i \sqrt{2} \gamma_{8}\left(m_{\pi}, 0, \ell\right)}{3 F_{0}^{2}}\right) \\
& 2 b_{\mathrm{I} 2}+\left.2 b_{\mathrm{II} 2}\right|_{\Lambda_{c}^{+} \leftarrow \Sigma_{c}^{*+}}=g_{2} g_{3} e\left(-\frac{i \gamma_{8}\left(m_{K}, \delta_{2}, \ell\right)}{\sqrt{2} F_{0}^{2}}-\frac{2 i \sqrt{2} \gamma_{8}\left(m_{\pi}, \delta_{2}, \ell\right)}{F_{0}^{2}}\right) \\
& +g_{4} g_{5} e\left(-\frac{i \gamma_{3}\left(m_{K}, 0, \ell\right)}{\sqrt{2} F_{0}^{2}}-\frac{i \gamma_{8}\left(m_{K}, 0, \ell\right)}{3 \sqrt{2} F_{0}^{2}}-\frac{2 i \sqrt{2} \gamma_{3}\left(m_{\pi}, 0, \ell\right)}{F_{0}^{2}}-\frac{2 i \sqrt{2} \gamma_{8}\left(m_{\pi}, 0, \ell\right)}{3 F_{0}^{2}}\right) \\
& 2 b_{\mathrm{I} 1}+\left.2 b_{\mathrm{II} 1}\right|_{\Xi_{c}^{+} \leftarrow \Xi_{c}^{* \prime+}}=g_{2} g_{3} e\left(-\frac{2 i \sqrt{2} \gamma_{3}\left(m_{K}, \delta_{2}, \ell\right)}{F_{0}^{2}}-\frac{2 i \sqrt{2} \gamma_{9}\left(m_{K}, \delta_{2}, \ell\right)}{F_{0}^{2}}-\frac{i \gamma_{3}\left(m_{\pi}, \delta_{2}, \ell\right)}{\sqrt{2} F_{0}^{2}}-\frac{i \gamma_{9}\left(m_{\pi}, \delta_{2}, \ell\right)}{\sqrt{2} F_{0}^{2}}\right) \\
& +g_{4} g_{5} e\left(\frac{4 i \sqrt{2} \gamma_{3}\left(m_{K}, 0, \ell\right)}{3 F_{0}^{2}}-\frac{2 i \sqrt{2} \gamma_{8}\left(m_{K}, 0, \ell\right)}{3 F_{0}^{2}}+\frac{i \sqrt{2} \gamma_{3}\left(m_{\pi}, 0, \ell\right)}{3 F_{0}^{2}}-\frac{i \gamma_{8}\left(m_{\pi}, 0, \ell\right)}{3 \sqrt{2} F_{0}^{2}}\right) \\
& 2 b_{\mathrm{I} 2}+\left.2 b_{\mathrm{II} 2}\right|_{\Xi_{c}^{+} \leftarrow \Xi_{c}^{* \prime+}}=g_{2} g_{3} e\left(-\frac{2 i \sqrt{2} \gamma_{8}\left(m_{K}, \delta_{2}, \ell\right)}{F_{0}^{2}}-\frac{i \gamma_{8}\left(m_{\pi}, \delta_{2}, \ell\right)}{\sqrt{2} F_{0}^{2}}\right) \\
& +g_{4} g_{5} e\left(-\frac{2 i \sqrt{2} \gamma_{3}\left(m_{K}, 0, \ell\right)}{F_{0}^{2}}-\frac{2 i \sqrt{2} \gamma_{8}\left(m_{K}, 0, \ell\right)}{3 F_{0}^{2}}-\frac{i \gamma_{3}\left(m_{\pi}, 0, \ell\right)}{\sqrt{2} F_{0}^{2}}-\frac{i \gamma_{8}\left(m_{\pi}, 0, \ell\right)}{3 \sqrt{2} F_{0}^{2}}\right) \\
& 2 b_{\mathrm{I} 1}+\left.2 b_{\mathrm{II} 1}\right|_{\Xi_{c}^{0} \leftarrow \Xi_{c}^{* \prime 0}}=g_{2} g_{3} e\left(-\frac{i \gamma_{3}\left(m_{K}, \delta_{2}, \ell\right)}{\sqrt{2} F_{0}^{2}}-\frac{i \gamma_{9}\left(m_{K}, \delta_{2}, \ell\right)}{\sqrt{2} F_{0}^{2}}+\frac{i \gamma_{3}\left(m_{\pi}, \delta_{2}, \ell\right)}{\sqrt{2} F_{0}^{2}}+\frac{i \gamma_{9}\left(m_{\pi}, \delta_{2}, \ell\right)}{\sqrt{2} F_{0}^{2}}\right) \\
& +g_{4} g_{5} e\left(\frac{i \sqrt{2} \gamma_{3}\left(m_{K}, 0, \ell\right)}{3 F_{0}^{2}}-\frac{i \gamma_{8}\left(m_{K}, 0, \ell\right)}{3 \sqrt{2} F_{0}^{2}}-\frac{i \sqrt{2} \gamma_{3}\left(m_{\pi}, 0, \ell\right)}{3 F_{0}^{2}}+\frac{i \gamma_{8}\left(m_{\pi}, 0, \ell\right)}{3 \sqrt{2} F_{0}^{2}}\right) \\
& 2 b_{\mathrm{I} 2}+\left.2 b_{\mathrm{II} 2}\right|_{\Xi_{c}^{0} \leftarrow \Xi_{c}^{* \prime 0}}=g_{2} g_{3} e\left(\frac{i \gamma_{8}\left(m_{\pi}, \delta_{2}, \ell\right)}{\sqrt{2} F_{0}^{2}}-\frac{i \gamma_{8}\left(m_{K}, \delta_{2}, \ell\right)}{\sqrt{2} F_{0}^{2}}\right) \\
& +g_{4} g_{5} e\left(-\frac{i \gamma_{3}\left(m_{K}, 0, \ell\right)}{\sqrt{2} F_{0}^{2}}-\frac{i \gamma_{8}\left(m_{K}, 0, \ell\right)}{3 \sqrt{2} F_{0}^{2}}+\frac{i \gamma_{3}\left(m_{\pi}, 0, \ell\right)}{\sqrt{2} F_{0}^{2}}+\frac{i \gamma_{8}\left(m_{\pi}, 0, \ell\right)}{3 \sqrt{2} F_{0}^{2}}\right)
\end{aligned}
$$

\section{THE DETERMINATION OF THE LOW ENERGY CONSTNATS (LECS)}

In principle, the low energy constants (LECs) in the chiral Lagrangians should be extracted through fitting to the experimental data. However, the available information on the heavy baryon system is still rather poor, which renders the extraction of the low energy constants $f, h$ nearly impossible. We try to estimate the LECs using the quark model. 
At the quark level, the flavor wave functions of the spin $\frac{1}{2}$ baryons can be written as follows.

$$
\left\{\begin{array} { l } 
{ \Lambda _ { c } ^ { + } = \frac { 1 } { \sqrt { 2 } } ( u d - d u ) c } \\
{ \Xi _ { c } ^ { + } = \frac { 1 } { \sqrt { 2 } } ( u s - s u ) c } \\
{ \Xi _ { c } ^ { 0 } = \frac { 1 } { \sqrt { 2 } } ( d s - s d ) c }
\end{array} \quad \left\{\begin{array}{l}
\Sigma_{c}^{++}=u u c \\
\Sigma_{c}^{+}=\frac{1}{\sqrt{2}}(u d+d u) c \\
\Sigma_{c}^{0}=d d c \\
\Xi_{c}^{\prime+}=\frac{1}{\sqrt{2}}(u s+s u) c \\
\Xi_{c}^{\prime 0}=\frac{1}{\sqrt{2}}(d s+s d) c \\
\Omega_{c}^{0}=s s c
\end{array}\right.\right.
$$

The flavor wave functions of the spin- $\frac{3}{2}$ baryons are similar.

The spin wave functions of the heavy baryons are

$$
\left\{\begin{array}{l}
\chi_{\frac{1}{2} \frac{1}{2}}=\frac{1}{\sqrt{2}}\left(\chi_{\frac{1}{2}} \chi_{-\frac{1}{2}} \chi_{\frac{1}{2}}-\chi_{-\frac{1}{2}} \chi_{\frac{1}{2}} \chi_{\frac{1}{2}}\right) \\
\chi_{\frac{1}{2}-\frac{1}{2}}=\frac{1}{\sqrt{2}}\left(\chi_{\frac{1}{2}} \chi_{-\frac{1}{2}} \chi_{-\frac{1}{2}}-\chi_{-\frac{1}{2}} \chi_{\frac{1}{2}} \chi_{-\frac{1}{2}}\right) \\
\chi_{\frac{1}{2} \frac{1}{2}}=\sqrt{\frac{2}{3}} \chi_{\frac{1}{2}} \chi_{\frac{1}{2}} \chi_{-\frac{1}{2}}-\frac{1}{\sqrt{6}}\left(\chi_{\frac{1}{2}} \chi_{-\frac{1}{2}} \chi_{\frac{1}{2}}+\chi_{-\frac{1}{2}} \chi_{\frac{1}{2}} \chi_{\frac{1}{2}}\right) \\
\chi_{\frac{1}{2}-\frac{1}{2}}=\frac{1}{\sqrt{6}}\left(\chi_{\frac{1}{2}} \chi_{-\frac{1}{2}} \chi_{-\frac{1}{2}}+\chi_{-\frac{1}{2}} \chi_{\frac{1}{2}} \chi_{-\frac{1}{2}}\right)-\sqrt{\frac{2}{3}} \chi_{-\frac{1}{2}} \chi_{-\frac{1}{2}} \chi_{\frac{1}{2}} \\
\chi_{\frac{3}{2} \frac{3}{2}}=\chi_{\frac{1}{2}} \chi_{\frac{1}{2}} \chi_{\frac{1}{2}} \\
\chi_{\frac{3}{2}-\frac{3}{2}}=\chi_{-\frac{1}{2}} \chi_{-\frac{1}{2}} \chi_{-\frac{1}{2}}
\end{array}\right.
$$

Comparing the matrix element of the same EM decay process at both the hadron and quark level, one can express the LECs in terms of the constituent quark masses and electric charge. For example, let's consider the $\Sigma_{c}^{+} \rightarrow \gamma+\Lambda_{c}^{+}$ decay process. At the hadron level, the Lagrangian is

$$
\mathcal{L}_{\Sigma_{c}^{+} \rightarrow \gamma+\Lambda_{c}^{+}}^{(2)}=-\frac{2 \sqrt{2}}{\Lambda_{\chi}} f_{2} e \bar{\Lambda}_{c}^{+} \sigma^{\mu \nu} \Sigma_{c}^{+} F_{\mu \nu}
$$

In the rest frame of the initial state, we choose the momentum of photon along the 3rd axis with the polarization along the $y$-axis. The matrix element of such a decay reads

$$
\left\langle\Lambda_{c}^{+},-\frac{1}{2}\left|\mathcal{L}_{y}\right| \Sigma_{c}^{+}, \frac{1}{2}\right\rangle=-\frac{4 \sqrt{2}}{\Lambda_{\chi}} f_{2} e
$$

where we have dropped the photon momentum and the image imaginary unit etc. These common factors also appear in the matrix element at the quark level. On the other hand, we consider the same matrix element at the quark level. The Gordon decomposition separates the QED interaction Lagrangian into the electric and magnetic parts. The light quark magnetic interaction reads

$$
\mathcal{L}_{\operatorname{Im}}=-\frac{1}{4} e\left(\frac{2}{3 m_{u}} \bar{u} \sigma^{\mu \nu} u-\frac{1}{3 m_{d}} \bar{d} \sigma^{\mu \nu} d-\frac{1}{3 m_{s}} \bar{s} \sigma^{\mu \nu} s\right) F_{\mu \nu}
$$

For the heavy quarks we have

$$
\mathcal{L}_{\operatorname{Im}, c}=-\frac{1}{4} e \frac{2}{3 m_{c}} \bar{c} \sigma^{\mu \nu} c F_{\mu \nu}, \quad \mathcal{L}_{\operatorname{Im}, b}=\frac{1}{4} e \frac{1}{3 m_{b}} \bar{b} \sigma^{\mu \nu} b F_{\mu \nu}
$$

respectively. We notice that the heavy quark magnetic interaction only contributes to the EM transitions between the $6^{*}$ and 6 -dimensional baryons. 
The flavor-spin wave functions of $\Lambda_{c}^{+}$and $\Sigma_{c}^{+}$at the quark level are

$$
\left\{\begin{array}{l}
\left|\Lambda_{c}^{+},-\frac{1}{2}\right\rangle=\frac{1}{\sqrt{2}}(|u d c\rangle-|d u c\rangle) \otimes\left[\frac{1}{\sqrt{2}}(|\uparrow \downarrow \downarrow\rangle-|\downarrow \uparrow \downarrow\rangle)\right] \\
\left|\Sigma_{c}^{+}, \frac{1}{2}\right\rangle=\frac{1}{\sqrt{2}}(|u d c\rangle+|d u c\rangle) \otimes\left[\sqrt{\frac{2}{3}}|\uparrow \uparrow \downarrow\rangle-\sqrt{\frac{1}{6}}(|\uparrow \downarrow \uparrow\rangle+|\downarrow \uparrow \uparrow\rangle)\right]
\end{array}\right.
$$

The magnetic interaction and tensor operator $\sigma^{\mu \nu}$ flips the spin of the up and down diquark system. The matrix element at the quark level reads

$$
\left\langle\Lambda_{c}^{+},-\frac{1}{2}\left|\mathcal{L}_{y}\right| \Sigma_{c}^{+}, \frac{1}{2}\right\rangle=-\frac{e}{3 \sqrt{3}}\left(\frac{1}{m_{u}}+\frac{1}{2 m_{d}}\right)
$$

With the commonly used values of the constituent quark masses [39].

$$
m_{u}=335.5 \mathrm{MeV}, m_{d}=339.5 \mathrm{MeV}, m_{s}=510 \mathrm{MeV}, m_{c}=1550 \mathrm{MeV}, m_{b}=4730 \mathrm{MeV},
$$

one obtains the value of the LEC $f_{2}$

$$
f_{2}=0.166
$$

The extraction of the other LECs for the $6^{*} \rightarrow 6$ process is more complicated. Let's take the decay channel $\Sigma_{c}^{*+} \rightarrow \Sigma_{c}^{+}+\gamma$ for an example. Both the $O\left(p^{2}\right)$ and $O\left(p^{3}\right)$ Lagrangians will contribute to the matrix element at the hadron level.

$$
\begin{gathered}
\left\langle\Sigma_{c}^{+}, \frac{1}{2}\left|\mathcal{L}^{(2)}\right| \Sigma_{c}^{*+}, \frac{3}{2}\right\rangle=\left(-\frac{4}{\sqrt{2} \Lambda_{\chi}^{2}} f_{3}-\frac{8}{\sqrt{2} \Lambda_{\chi}^{2}} \tilde{f}_{3}\right) e M_{\Sigma_{c}^{*+}} \\
\left\langle\Sigma_{c}^{+}, \frac{1}{2}\left|\mathcal{L}^{(3)}\right| \Sigma_{c}^{*+}, \frac{3}{2}\right\rangle=\left(-\frac{4\left(h_{13}+\tilde{h}_{13}\right)}{\sqrt{2} \Lambda_{\chi}^{3}}+\frac{4\left(h_{23}+\tilde{h}_{23}\right)}{\Lambda_{\chi}^{3}}\right) e\left(M_{\Sigma_{c}^{*+}}+M_{\Sigma_{c}^{+}}\right)\left(M_{\Sigma_{c}^{*+}}-M_{\Sigma_{c}^{+}}\right)
\end{gathered}
$$

The matrix element at the quark level reads

$$
\left\langle\Sigma_{c}^{+}, \frac{1}{2}|\mathcal{L}| \Sigma_{c}^{*+}, \frac{3}{2}\right\rangle=-\frac{e}{3 \sqrt{6}}\left(\frac{1}{m_{u}}-\frac{1}{2 m_{d}}-\frac{1}{m_{c}}\right)
$$

There are similar equations at both the hadron and quark level for all the other $6^{*} \rightarrow 6$ processes.

At $O\left(p^{2}\right)$, there are only two unknown LECs $f_{3}$ and $\tilde{f}_{3}$ while there are more equations. The best fit in the $\mathrm{SU}(3)$ flavor symmetry limit leads to

$$
f_{3}=0.099, \quad \tilde{f}_{3}=-0.037 .
$$

In the extraction of the $O\left(p^{3}\right)$ LECs $h_{13}$ etc, we use the above values as input and take into account the explicit $\mathrm{SU}(3)$ symmetry breaking, i.e., using the experimental values of the heavy baryon masses and different values for $m_{u}$ and $m_{s}$. Solving these above equations simultaneously, we get the values of the LECs at $O\left(p^{3}\right)$

$$
h_{13}=0.073, \quad h_{23}=0.034, \quad \tilde{h}_{13}=0.035, \quad \tilde{h}_{23}=0.061
$$

Similarly, we can extract the LECs for the $6^{*} \rightarrow \overline{3}$ process.

$$
f_{4}=0.133, \quad h_{46}=0.056, \quad h_{56}=-0.039
$$

All the values of LECs are collected in Table 
TABLE III: The values of the dimensionless LECs for the charmed baryon systems from the quark model.

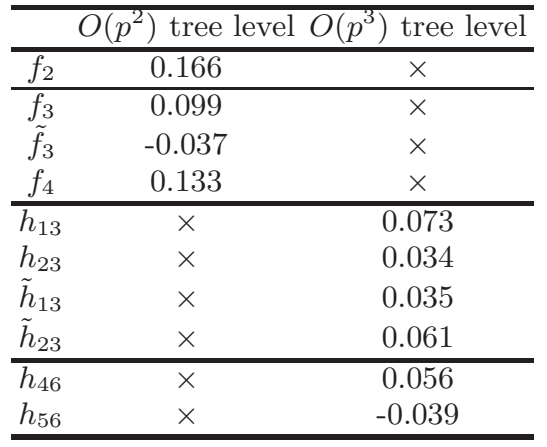

TABLE IV: The EM decay width of the charmed baryons in unit of keV.

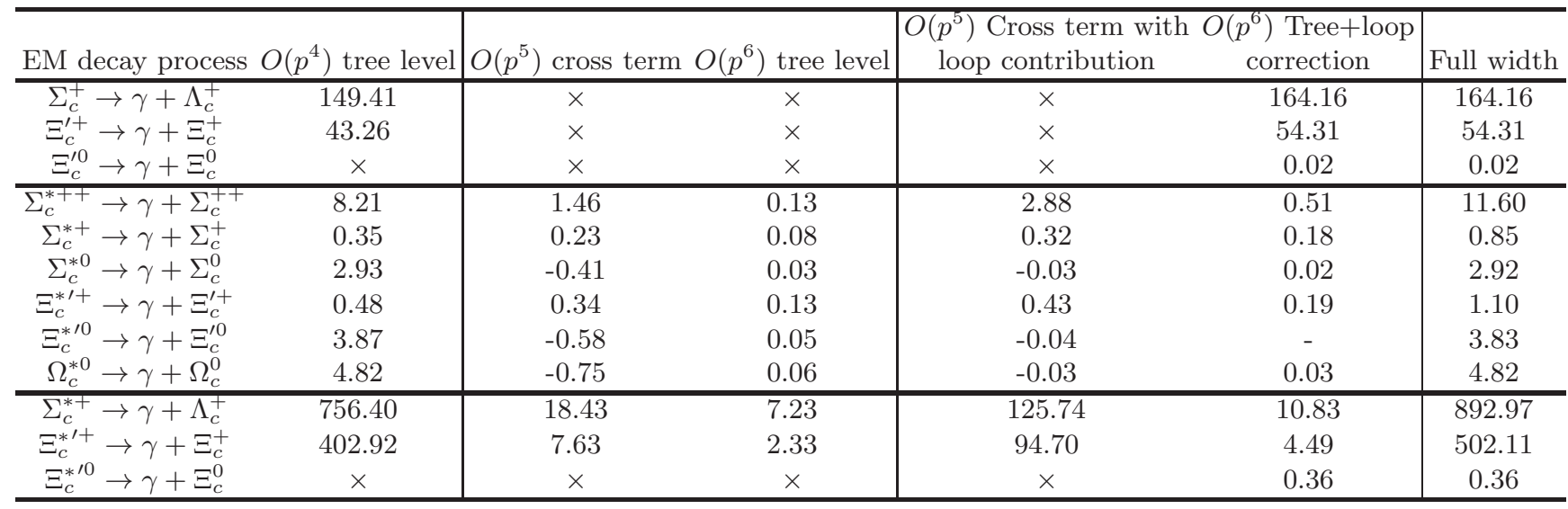

\section{NUMERICAL RESULTS AND DISCUSSIONS}

So far, all LECs and the chiral loop corrections have been obtained. The EM decay width reads

$$
\Gamma=\frac{\ell}{8 \pi M_{A}^{2}}|\mathcal{M}|^{2}
$$

We collect the numerical results of the EM decay widths of the ground state charmed baryons in Table IV (in unit of $\mathrm{keV})$. The notation " $\times$ " means no contribution in this channel and "-" means the contribution is strongly suppressed. In Table IV the first three rows, middle six rows and the last three rows correspond to the $6 \rightarrow \overline{3}, 6^{*} \rightarrow 6$ and $6^{*} \rightarrow \overline{3}$ EM decays respectively.

The EM decay width is proportional to the cubic of the photon momentum, which depends on the mass difference of the initial and final heavy baryons. The larger the mass difference, the larger the decay width. Therefore the $6^{*} / 6 \rightarrow \overline{3}$ EM decay widths may reach several hundred $\mathrm{keV}$ while the $6^{*} \rightarrow 6$ transition width is only a few $\mathrm{keV}$.

From Table IV, the numerical results of the EM decay widths show very good convergence of the chiral expansion. For the $6 \rightarrow \overline{3}$ decays, the leading order widths are much larger than the loop contributions. For the nine spin- $\frac{3}{2}$ to $\frac{1}{2}$ transitions, the leading order amplitude yields the EM decay width at $O\left(p^{4}\right)$. The cross term from the tree-level $O\left(p^{2}\right)$ and $O\left(p^{3}\right)$ amplitudes contributes to the decay width at $O\left(p^{5}\right)$ while the $O\left(p^{3}\right)$ amplitude alone contributes to the widths at $O\left(p^{6}\right)$. From the second, third and fourth column of Table IV the numerical results of the $O\left(p^{4}\right)$, $O\left(p^{5}\right)$ and $O\left(p^{6}\right)$ corrections decrease accordingly, consistent with the chiral power. When the loop contributions are added in the fifth and sixth columns, the numerical values also maintain the same decreasing trend.

A remarkable feature in Table [V is the strong suppression of the $\Xi_{c}^{\prime 0} \rightarrow \gamma+\Xi_{c}^{0}$ and $\Xi_{c}^{* \prime 0} \rightarrow \gamma+\Xi_{c}^{0}$ decays. As we have pointed out before, the exact $\mathrm{SU}(3)$ U-spin flavor symmetry forbids the two neutral EM decay processes $\Xi_{c}^{\prime 0} \rightarrow \gamma+\Xi_{c}^{0}$ and $\Xi_{c}^{* \prime 0} \rightarrow \gamma+\Xi_{c}^{0}$. There do not exist tree-level contributions at $O\left(p^{2}\right)$ and $O\left(p^{3}\right)$. The non-zero tree-level contribution to the decay amplitude starts at $O\left(p^{4}\right)$ which explicitly breaks the $\mathrm{SU}(3)$ flavor symmetry through the current quark mass matrix. On the other hand, the chiral loop corrections appear at $O\left(p^{3}\right)$ if we use the physical mass for the eight Goldstone bosons. In fact, the chiral corrections from the pion, kaon and eta mesons 
will cancel exactly at $O\left(p^{3}\right)$ if their masses and decay constants are the same, which is also required by the U-spin symmetry. From Table IV we do notice that the numerical values of the loop contributions of these two channels are indeed much smaller than their counterparts in the same multiplet.

In short summary, we have investigated the electromagnetic decays of the heavy baryon systems within the heavy baryon chiral perturbation theory. After introducing the leading order chiral Lagrangians and constructing the $O\left(p^{2}\right)$ and $O\left(p^{3}\right)$ Lagrangians, we have systematically calculated the twelve EM decay amplitudes up to $O\left(p^{3}\right)$. With the help of the quark model, we estimate all the low energy constants at $O\left(p^{2}\right)$ and $O\left(p^{3}\right)$. The convergence of the chiral expansion is quite good.

In the future, the EM decay widths of the charmed and bottomed baryons may be measured at facilities such as LHCb and JPARC. We sincerely hope our present work will be useful to future experiments. Moreover, the explicit chiral structures of the heavy baryon decay amplitudes derived in this work may also be useful to the possible chiral extrapolations of the future lattice simulations of these EM decay amplitudes.

\section{ACKNOWLEDGMENTS}

N.J. is very grateful to L. Ma, G.-J. Wang and Professor W. Z. Deng for very helpful discussions. This project is supported by the National Natural Science Foundation of China under Grant No. 11261130311. 


\section{Appendix}

\section{EM DECAY WIDTHS OF THE BOTTOMED BARYONS}

In the same framework, we estimate the EM decay widths of the bottomed baryons. The chiral Lagrangians of the bottomed baryons are the same as those for the charmed baryons. All the coefficients $f^{b}, h^{b}$ are collected in Table II. They can also be determined from the quark model. The value of the LECs are listed in Table $\mathbf{V}$

TABLE V: The dimensionless LECs for the bottomed baryon systems.

\begin{tabular}{|c|c|c|}
\hline & $\overline{O\left(p^{2}\right) \text { tree level }}$ & $O\left(p^{3}\right)$ tree level \\
\hline$f_{2}^{b}$ & 0.166 & $\times$ \\
\hline$f_{3}^{b}$ & 0.047 & $\times$ \\
\hline$\tilde{f}_{3}^{b}$ & -0.021 & $\times$ \\
\hline$f_{4}^{b}$ & 0.058 & $\times$ \\
\hline$\overline{h_{13}^{b}}$ & $\times$ & 0.369 \\
\hline$h_{23}^{b}$ & $x$ & -0.058 \\
\hline$\tilde{h}_{13}^{b}$ & $\times$ & -0.096 \\
\hline$\tilde{h}_{23}^{b}$ & $\times$ & 0.068 \\
\hline$\overline{h_{46}^{b}}$ & $\times$ & 0.087 \\
\hline$h_{56}^{b}$ & $x$ & -0.061 \\
\hline
\end{tabular}

At present, the experimental data on the bottomed baryons is scarce. We choose the average value of the bottomed baryon mass as $M_{6}^{b}=5891.87 \mathrm{MeV}[1]$. We naively assume that the the mass splitting between 6 and $6^{*}$-dimensional is inversely proportional to the heavy quark mass.

$$
\left(M_{6^{*}}^{b}-M_{6}^{b}\right) m_{b}=\left(M_{6^{*}}^{c}-M_{6}^{c}\right) m_{c}
$$

Then we obtain the mass splitting for the bottomed baryons as

$$
\delta_{2}^{b}=21.97 \mathrm{MeV}
$$

We let $\delta_{1}^{b}, \delta_{3}^{b}$ take roughly the same values as in the charmed baryon systems since these splittings arise from the strong interaction of the light diquark system. The above values will be used in the calculation of the chiral loop corrections.

Since the EM decay widths are very sensitive to the photon momentum, we explicitly keep the photon momentum $\ell$ in the decay width formula which shall be measured by future experiments. The EM decay widths of the bottomed baryons are listed below in unit of $\mathrm{keV}$ while the photon momentum $\ell$ is in unit of $\mathrm{MeV}$.

$$
\begin{aligned}
\Gamma_{\Sigma_{b}^{0} \rightarrow \gamma+\Lambda_{b}^{0}} & =4.15 \times 10^{-5} \ell^{3} \\
\Gamma_{\Xi_{b}^{\prime 0} \rightarrow \gamma+\Xi_{b}^{0}} & =4.67 \times 10^{-5} \ell^{3} \\
\Gamma_{\Xi_{b}^{\prime-} \rightarrow \gamma+\Xi_{b}^{-}} & =2.18 \times 10^{-8} \ell^{3} \\
\Gamma_{\Sigma_{b}^{*+} \rightarrow \gamma+\Sigma_{b}^{+}} & =5.37 \times 10^{-5} \ell^{3}+5.74 \times 10^{-7} \ell^{4}+3.20 \times 10^{-9} \ell^{5} \\
\Gamma_{\Sigma_{b}^{* 0} \rightarrow \gamma+\Sigma_{b}^{0}} & =5.12 \times 10^{-6} \ell^{3}+3.21 \times 10^{-8} \ell^{4}+2.89 \times 10^{-10} \ell^{5} \\
\Gamma_{\Sigma_{b}^{*-} \rightarrow \gamma+\Sigma_{b}^{-}} & =7.86 \times 10^{-6} \ell^{3}+13.10 \times 10^{-8} \ell^{4}+14.22 \times 10^{-10} \ell^{5} \\
\Gamma_{\Xi_{b}^{* \prime \prime} \rightarrow \gamma+\Xi_{b}^{\prime 0}} & =5.30 \times 10^{-6} \ell^{3}+2.98 \times 10^{-8} \ell^{4}+2.14 \times 10^{-10} \ell^{5} \\
\Gamma_{\Xi_{b}^{* \prime} \rightarrow \gamma+\Xi_{b}^{\prime-}} & =7.86 \times 10^{-6} \ell^{3}+13.82 \times 10^{-8} \ell^{4}+13.64 \times 10^{-10} \ell^{5} \\
\Gamma_{\Omega_{b}^{*-} \rightarrow \gamma+\Omega_{b}^{-}} & =8.29 \times 10^{-6} \ell^{3}+15.59 \times 10^{-8} \ell^{4}+26.86 \times 10^{-10} \ell^{5} \\
\Gamma_{\Sigma_{b}^{* 0} \rightarrow \gamma+\Lambda_{b}^{0}} & =7.55 \times 10^{-5} \ell^{3}-1.85 \times 10^{-7} \ell^{4}+2.31 \times 10^{-10} \ell^{5} \\
\Gamma_{\Xi_{b}^{* \prime \prime} \rightarrow \gamma+\Xi_{b}^{0}} & =7.95 \times 10^{-5} \ell^{3}-3.13 \times 10^{-7} \ell^{4}+2.84 \times 10^{-10} \ell^{5} \\
\Gamma_{\Xi_{b}^{* \prime}-\rightarrow \gamma+\Xi_{b}^{-}} & =2.15 \times 10^{-11} \ell^{5}
\end{aligned}
$$

We also list the numerical results of the EM decay widths in TableVI where the masses of the initial and final state bottom baryons are known experimentally [1]. 
TABLE VI: The EM decay widths of the bottomed baryons decay in unit of keV.

\begin{tabular}{|c|c|c|c|c|}
\hline EM decay process & $O\left(p^{4}\right)$ tree level & $\begin{array}{c}O\left(p^{5}\right) \text { Cross term witl } \\
\text { loop contribution }\end{array}$ & $\begin{array}{c}O\left(p^{6}\right) \text { Tree+loop } \\
\text { correction }\end{array}$ & Full width \\
\hline$\Sigma_{b}^{0} \rightarrow \gamma+\Lambda_{b}^{0}$ & 255.90 & $x$ & 287.65 & 287.65 \\
\hline$\Sigma_{b}^{*+} \rightarrow \gamma+\Sigma_{b}^{+}$ & 0.48 & 0.11 & 0.01 & 0.60 \\
\hline$\Sigma_{b}^{* 0} \rightarrow \gamma+\Sigma_{b}^{0}$ & 0.04 & 0.01 & - & 0.05 \\
\hline$\Sigma_{b}^{*-} \rightarrow \gamma+\Sigma_{b}^{-}$ & 0.06 & 0.02 & - & 0.08 \\
\hline$\Sigma_{b}^{* 0} \rightarrow \gamma+\Lambda_{b}^{0}$ & 701.63 & -361.29 & 94.83 & 435.17 \\
\hline$\Xi_{b}^{* \prime 0} \rightarrow \gamma+\Xi_{b}^{0}$ & 281.32 & -168.70 & 23.34 & 135.96 \\
\hline$\Xi_{b}^{* \prime-} \rightarrow \gamma+\Xi_{b}^{-}$ & $\times$ & $\times$ & 1.87 & 1.87 \\
\hline
\end{tabular}

\section{INTEGRALS AND FUNCTIONS}

1. The integral with one meson line

$$
I(m)=\frac{m^{2}}{16 \pi^{2}}\left(R+\ln \frac{m^{2}}{\mu^{2}}\right)
$$

2. The integral with one meson line and one baryon line

$$
J(m, \omega)=\frac{1}{8 \pi^{2}}\left[\omega(R-1)+\omega \ln \frac{m^{2}}{\mu^{2}}+K\right], \omega=v \cdot k+\delta
$$

and

$$
K=\left\{\begin{array}{l}
2 \sqrt{\omega^{2}-m^{2}} \operatorname{arccosh} \frac{\omega}{m}-2 i \pi \sqrt{\omega^{2}-m^{2}}, \quad \omega>m \\
-2 \sqrt{\omega^{2}-m^{2}} \operatorname{arccosh} \frac{-\omega}{m}, \quad \omega<-m \\
2 \sqrt{m^{2}-\omega^{2}} \arccos \frac{-\omega}{m}, \quad \omega^{2}<m^{2}
\end{array}\right.
$$

3. The integral with two meson lines and one baryon line

3.1 scaler integral

$$
L=\frac{1}{8 \pi^{2} v \cdot l}\left\{\begin{array}{c}
\frac{1}{i} \int \frac{d^{d} q}{(2 \pi)^{d}} \frac{1}{\left(m^{2}-(q+l)^{2}\right)\left(m^{2}-q^{2}\right)[v \cdot q+\omega]}, \quad l^{2}=0 \\
-\frac{1}{2}\left[\left(\operatorname{arccosh} \frac{-\omega+v \cdot l}{m}\right)^{2}-\left(\operatorname{arccosh} \frac{-\omega}{m}\right)^{2}\right] \\
+\pi i\left[\ln \left(\sqrt{(\omega-v \cdot l)^{2}-m^{2}}+\omega-v \cdot l\right)-\ln \left(\sqrt{\omega^{2}-m^{2}}+\omega\right)\right], \quad \beta>m \\
\frac{1}{2}\left[\left(\operatorname{arccosh} \frac{-\omega+v \cdot l}{m}\right)^{2}-\left(\operatorname{arccosh} \frac{-\omega}{m}\right)^{2}\right], \quad \beta<-m \\
\frac{1}{2}\left[\left(\arccos \frac{-\omega+v \cdot l}{m}\right)^{2}-\left(\arccos \frac{-\omega}{m}\right)^{2}\right], \quad \beta^{2}<m^{2}
\end{array}\right.
$$

where $\beta=\omega-v \cdot l$

3.2 vector integral

$$
\begin{gathered}
L_{\mu}=\frac{1}{i} \int \frac{d^{d} q}{(2 \pi)^{d}} \frac{q_{\mu}}{\left(m^{2}-(q+l)^{2}\right)\left(m^{2}-q^{2}\right)[v \cdot q+\omega]}=\gamma_{1} l_{\mu}+\gamma_{2} v_{\mu} \\
\gamma_{1}(m, \omega, v \cdot l)=\frac{1}{v \cdot l}\left(I_{2}(m)-\omega L(m, \omega, v \cdot l)\right)-\frac{1}{2(v \cdot l)^{2}}[J(m, \beta)-J(m, \omega)]
\end{gathered}
$$




$$
\gamma_{2}(m, \omega, v \cdot l)=\frac{1}{2(v \cdot l)^{2}}[J(m, \beta)-J(m, \omega)]
$$

in the rest frame, $v \cdot l=\ell$, which is the modular of photon 3-momentum.

3.3 Second-order integral

$$
\begin{gathered}
L_{\mu \nu}=\frac{1}{i} \int \frac{d^{d} q}{(2 \pi)^{d}} \frac{q_{\mu} q_{\nu}}{\left(m^{2}-(q+l)^{2}\right)\left(m^{2}-q^{2}\right)[v \cdot q+\omega]} \\
=\gamma_{3} g_{\mu \nu}+\gamma_{4} l_{\mu} l_{\nu}+\gamma_{5} v_{\mu} l_{\nu}+\gamma_{6} l_{\mu} v_{\nu}+\gamma_{7} v_{\mu} v_{\nu} \\
\gamma_{3}(m, \omega, \ell)=-\frac{1}{2(d-2) \ell}\left\{\omega J(m, \omega)+(\beta-2 \omega+2 \ell) J(m, \beta)-2 m^{2} \ell L(m, \omega, \ell)\right\} \\
\gamma_{4}(m, \omega, \ell)=\frac{1}{2(d-2) \ell^{3}}\{(\omega-d \omega) J(m, \omega)+J(m, \beta)(-(d-1)(\beta-2 \omega)-2 \ell) \\
\gamma_{5,6}(m, \omega, \ell)= \\
\left.+2 \ell\left((d-2) \omega^{2}+m^{2}\right) L(m, \omega, \ell)-(d-2) \ell I_{2}(m)(2 \omega+\ell)\right\} \\
\gamma_{7}(m, \omega, \ell)= \\
\frac{1}{2(d-2) \ell^{2}}\left\{J(m, \beta)(\beta(d-1)-d \omega+2 \ell)+\omega J(m, \omega)-2 m^{2} \ell L(m, \omega, \ell)\right\}
\end{gathered}
$$

$d$ arises from the dimensional regularization in d-dimensional space.

3.4 Third-order integral

$$
\begin{aligned}
L_{\mu \alpha \beta}= & \frac{1}{i} \int \frac{d^{d} q}{(2 \pi)^{d}} \frac{q_{\mu} q_{\alpha} q_{\beta}}{\left(m^{2}-(q+l)^{2}\right)\left(m^{2}-q^{2}\right)[v \cdot q+\omega]} \\
= & \gamma_{8} g_{\mu \alpha} l_{\beta}+\gamma_{9} g_{\mu \beta} l_{\alpha}+\gamma_{10} g_{\alpha \beta} l_{\mu} \\
& +\gamma_{11} g_{\mu \alpha} v_{\beta}+\gamma_{12} g_{\mu \beta} v_{\alpha}+\gamma_{13} g_{\alpha \beta} v_{\mu} \\
& +\gamma_{14} l_{\mu} l_{\alpha} l_{\beta}+\gamma_{15} l_{\mu} l_{\alpha} v_{\beta}+\gamma_{16} l_{\mu} v_{\alpha} l_{\beta}+\gamma_{17} v_{\mu} l_{\alpha} l_{\beta} \\
& +\gamma_{18} l_{\mu} v_{\alpha} v_{\beta}+\gamma_{19} v_{\mu} l_{\alpha} v_{\beta}+\gamma_{20} v_{\mu} v_{\alpha} l_{\beta}+\gamma_{21} v_{\mu} v_{\alpha} v_{\beta}
\end{aligned}
$$

For completeness, we list all the functions $\gamma_{i}$ as follows, although only $\gamma_{8}$ and $\gamma_{9}$ are used in the calculations of loop-f. Some functions may be useful in the calculation of the magnetic moment of heavy baryons.

$$
\begin{aligned}
\gamma_{8,9,10}(m, \omega, \ell)= & \frac{1}{2(d-2)(d-1) \ell^{2}}\left\{-\left(\beta^{2}+(d-2) m^{2}\right) J(m, \beta)\right. \\
& \left.+J(m, \omega)\left((d-2) m^{2}+\omega(-d \ell+\omega+\ell)\right)+\ell\left(2(d-1) m^{2}\left(I_{2}(m)-L \omega\right)+(d-2) I(m)\right)\right\} \\
\gamma_{11,12,13}(m, \omega, \ell)= & \frac{1}{2(d-2)(d-1) \ell}\left\{J(m, \beta)\left((d-2) m^{2}+\beta(2(d-1) \ell-\beta(d-2))\right)\right. \\
& \left.+J(m, \omega)\left(-(d-2) m^{2}+(d-2) \omega^{2}-2(d-1) \omega \ell\right)-(d-2) \ell I(m)\right\} \\
\gamma_{14}(m, \omega, \ell)= & \frac{1}{6(d-2)(d-1) d \ell^{4}}\left\{-3 d J(m, \beta)\left(\beta^{2}((d-2) d+3)+(d-2)\left(3(d-1) \ell^{2}+2 m^{2}\right)\right)\right. \\
& +3 d J(m, \omega)\left(2(d-2) m^{2}+\omega\left(((d-2) d+3) \omega-3(d-1)^{2} \ell\right)\right) \\
& -6(d-1) d L \omega \ell\left((d-2) \omega^{2}+3 m^{2}\right)+(d-1) \ell I_{2}(m)\left(12(2 d-1) m^{2}+(d-2) d\left(6 \omega^{2}+2 \ell^{2}+3 \omega \ell\right)\right) \\
& +6(d-2)((d-1) d+1) \ell I(m)\} \\
\gamma_{15,16,17}(m, \omega, \ell)= & \frac{1}{2(d-2)(d-1) \ell^{3}}\left\{2\left(\ell\left(2(d-1) m^{2}\left(L \omega-I_{2}(m)\right)-(d-2) I(m)\right)-\left((d-2) m^{2}+\omega^{2}\right) J(m, \omega)\right)\right. \\
& \left.+J(m, \beta)\left(2 \beta^{2}+(d-2)\left((d-1) \ell^{2}+2 m^{2}\right)+2 \beta(d-1) \ell\right)\right\} \\
\gamma_{18,19,20}(m, \omega, \ell)= & \frac{1}{2(d-2)(d-1) \ell^{2}}\left\{J(m, \omega)\left(\left(d^{2}+d-2\right) \omega \ell+(d-2) m^{2}-(d-2) \omega^{2}\right)\right. \\
& \left.+J(m, \beta)\left(\beta(\beta(d-2)-4(d-1) \ell)-(d-2) m^{2}\right)+(d-2)^{2}(-\ell) I(m)\right\} \\
\gamma_{21}(m, \omega, \ell)= & \frac{1}{2(d-1) \ell}\left\{\left(\beta^{2} d-m^{2}\right) J(m, \beta)+\left(m^{2}-d \omega^{2}\right) J(m, \omega)+d \ell I(m)\right\}
\end{aligned}
$$


[1] K. A. Olive et al. [Particle Data Group Collaboration], Chin. Phys. C 38, 090001 (2014).

[2] F. Wick [CDF Collaboration], Int. J. Mod. Phys. Conf. Ser. 02, 163 (2011) arXiv:1105.0517 [hep-ex]].

[3] T. Aaltonen et al. [CDF Collaboration], Phys. Rev. D 84, 012003 (2011) arXiv:1105.5995 [hep-ex]].

[4] T. Uppal and R. C. Verma, Phys. Rev. D 47, 2858 (1993).

[5] A. Majethiya, B. Patel and P. C. Vinodkumar, arXiv:0902.2536 [hep-ph].

[6] S. J. Puglia, M. J. Ramsey-Musolf and S. L. Zhu, Phys. Rev. D 63, 034014 (2001) hep-ph/0008140.

[7] U. G. Meissner and S. Steininger, Nucl. Phys. B 499, 349 (1997) hep-ph/9701260.

[8] L. S. Geng, J. Martin Camalich and M. J. Vicente Vacas, Phys. Lett. B 676, 63 (2009) arXiv:0903.0779 [hep-ph]].

[9] S. L. Zhu and Y. B. Dai, Phys. Rev. D 59, 114015 (1999) hep-ph/9810243.

[10] H. Y. Cheng, C. Y. Cheung, G. L. Lin, Y. C. Lin, T. M. Yan and H. L. Yu, Phys. Rev. D 47, 1030 (1993) hep-ph/9209262.

[11] H. Y. Cheng, C. Y. Cheung, G. L. Lin, Y. C. Lin, T. M. Yan and H. L. Yu, Phys. Rev. D 49, 5857 (1994) [Phys. Rev. D 55, 5851 (1997)] hep-ph/9312304.

[12] H. W. K. Cheung, In *Les Arcs 1993, Proceedings, QCD and high energy hadronic interactions* 473-477. and Fermilab Batavia - FERMILAB-Conf-93-164 (93/06,rec.Aug.) 5 p. C

[13] H. Bahtiyar, K. U. Can, G. Erkol and M. Oka, arXiv:1503.07361 [hep-lat].

[14] M. C. Banuls, A. Pich and I. Scimemi, Phys. Rev. D 61, 094009 (2000) hep-ph/9911502.

[15] J. G. Korner, M. Kramer and D. Pirjol, Prog. Part. Nucl. Phys. 33, 787 (1994) hep-ph/9406359.

[16] P. L. Cho, Phys. Rev. D 50, 3295 (1994) hep-ph/9401276].

[17] M. J. Savage, Phys. Lett. B 345, 61 (1995) hep-ph/9408294.

[18] M. A. Ivanov, J. G. Korner and V. E. Lyubovitskij, Phys. Lett. B 448, 143 (1999) hep-ph/9811370.

[19] M. A. Ivanov, J. G. Korner, V. E. Lyubovitskij and A. G. Rusetsky, Phys. Rev. D 60, 094002 (1999) hep-ph/9904421.

[20] S. Tawfiq, J. G. Korner and P. J. O’Donnell, Phys. Rev. D 63, 034005 (2001) hep-ph/9909444.

[21] N. Jiang, X. L. Chen and S. L. Zhu, Phys. Rev. D 90, no. 7, 074011 (2014) arXiv:1403.5404 [hep-ph]].

[22] S. Weinberg, Physica A 96, 327 (1979).

[23] J. Gasser and H. Leutwyler, Annals Phys. 158, 142 (1984).

[24] J. Gasser and H. Leutwyler, Nucl. Phys. B 250, 465 (1985).

[25] J. Gasser, M. E. Sainio and A. Svarc, Nucl. Phys. B 307, 779 (1988).

[26] E. E. Jenkins and A. V. Manohar, Phys. Lett. B 255, 558 (1991).

[27] V. Bernard, N. Kaiser, J. Kambor and U. G. Meissner, Nucl. Phys. B 388, 315 (1992).

[28] V. Bernard, N. Kaiser and U. G. Meissner, Nucl. Phys. B 383, 442 (1992).

[29] S. Scherer, Adv. Nucl. Phys. 27, 277 (2003) hep-ph/0210398].

[30] V. Bernard, N. Kaiser and U. -G. Meissner, Int. J. Mod. Phys. E 4, 193 (1995) hep-ph/9501384.

[31] N. Fettes, U. -G. Meissner, M. Mojzis and S. Steininger, Annals Phys. 283, 273 (2000) [Erratum-ibid. 288, 249 (2001)] hep-ph/0001308.

[32] V. Bernard, N. Kaiser and U. -G. Meissner, Nucl. Phys. A 615, 483 (1997) hep-ph/9611253.

[33] M. Mojzis, Eur. Phys. J. C 2, 181 (1998) hep-ph/9704415.

[34] N. Fettes, U. -G. Meissner and S. Steininger, Nucl. Phys. A 640, 199 (1998) hep-ph/9803266.

[35] N. Fettes and U. -G. Meissner, Nucl. Phys. A 693, 693 (2001) hep-ph/0101030.

[36] K. Johnson and E. C. G. Sudarshan, Annals Phys. 13, 126 (1961).

[37] T. -M. Yan, H. -Y. Cheng, C. -Y. Cheung, G. -L. Lin, Y. C. Lin and H. -L. Yu, Phys. Rev. D 46, 1148 (1992) [Erratum-ibid. D 55, $5851(1997)]$.

[38] S. Pokorski, Cambridge, Uk: Univ. Pr. ( 1987) 394 P. ( Cambridge Monographs On Mathematical Physics)

[39] M. D. Scadron, R. Delbourgo and G. Rupp, J. Phys. G 32, 735 (2006) hep-ph/0603196. 